\title{
Global effective-field-theory analysis of new-physics effects in (semi)leptonic kaon decays
}

\author{
Martín González-Alonso $^{a}$ and Jorge Martin Camalich ${ }^{b}$ \\ ${ }^{a}$ IPN de Lyon/CNRS, Universite Lyon 1, \\ Villeurbanne, France \\ ${ }^{b}$ CERN, Theoretical Physics Department, \\ Geneva, Switzerland \\ E-mail: mgonzalez@ipnl.in2p3.fr, jorge.martin.camalich@cern.ch
}

Abstract: We analyze the decays $K \rightarrow \pi \ell \nu$ and $P \rightarrow \ell \nu(P=K, \pi, \ell=e, \mu)$ using a low-energy Effective-Field-Theory approach to parametrize New Physics and study the complementarity with baryon $\beta$ decays. We then provide a road map for a global analysis of the experimental data, with all the Wilson coefficients simultaneously, and perform a fit leading to numerical bounds for them and for $V_{u s}$. A prominent result of our analysis is a reinterpretation of the well-known $V_{u d}-V_{u s}$ diagram as a strong constraint on new physics. Finally, we reinterpret our bounds in terms of the $\mathrm{SU}(2)_{L} \times \mathrm{U}(1)_{Y}$-invariant operators, provide bounds to the corresponding Wilson coefficients at the $\mathrm{TeV}$ scale and compare our results with collider searches at the LHC.

Keywords: Beyond Standard Model, Effective field theories, Kaon Physics

ARXIV EPRINT: 1605.07114 


\section{Contents}

1 Introduction 1

2 The low-energy effective Lagrangian 3

2.1 Renormalization and scale running of the Wilson coefficients 4

3 Decay observables 5

$3.1 \pi_{\ell 2(\gamma)}$ and $K_{\ell 2(\gamma)}$

$\begin{array}{lll}3.2 & P_{\ell 2 \gamma} & 7\end{array}$

$\begin{array}{lll}3.3 & K_{\ell 3(\gamma)} & 7\end{array}$

3.3.1 Kinematical distribution 8

3.3.2 Total rates 9

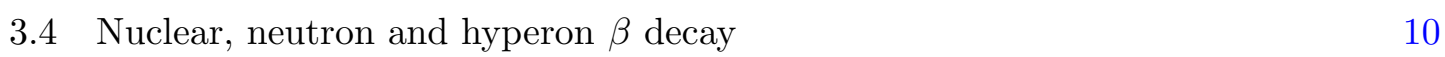

4 Strategy for the global analyses $\quad 12$

$\begin{array}{ll}4.1 \text { Quadratic contributions of the WC } & 14\end{array}$

5 Phenomenology 16

$\begin{array}{lll}5.1 & \text { Inputs } & 16\end{array}$

$\begin{array}{lll}5.1 .1 & \text { Experimental } & 16\end{array}$

$\begin{array}{lll}5.1 .2 & \text { Theoretical } & 18\end{array}$

$\begin{array}{lll}5.2 & \text { Fit } & 19\end{array}$

5.2.1 The $V_{u d}-V_{u s}$ plane revamped $\quad 21$

5.3 Minimal Flavor Violation and SM limits 21

6 SMEFT and complementarity with collider searches $\quad 24$

6.1 RGE running and matching to the SMEFT 24

6.2 MFV limit 26

$\begin{array}{lll}6.3 & \text { Bounds on scalar and tensor interactions } & 26\end{array}$

$\begin{array}{llr}7 & \text { Conclusions } & 29\end{array}$

$\begin{array}{ll}\text { A WC bounds at } \mu=1 \mathrm{TeV} & 30\end{array}$

\section{Introduction}

The $K \rightarrow \pi \ell \nu\left(K_{\ell 3}\right)$ and $P \rightarrow \ell \nu\left(P_{\ell 2}\right)$ decays, where $P=\pi, K$ and $\ell=e, \mu$, boast one of the most precise data bases in hadronic weak decays [1-4]. The hadronic form factors necessary to describe these processes are flagship quantities for lattice QCD (LQCD) and the theoretical accuracy at which they are calculated is now below the percent level (relative 
uncertainty) $[5,6]$. Much work has also been done in Chiral Perturbation Theory (ChPT) and using dispersive methods to understand analytically low-energy theorems and small contributions to the decay rates such as isospin breaking and the electromagnetic radiative corrections [7-21]. This makes $P_{\ell 2}$ and $K_{\ell 3}$ ideal flavor benchmarks to test the CabibboKobayashi-Maskawa (CKM) structure in the Standard Model (SM) and to search for new physics (NP). In fact, a systematic search of NP effects in (semi)leptonic kaon decays is particularly interesting at the moment as several anomalies have been recently reported in flavor observables such as $B$ decay rates [22-29] or $K \bar{K}$ mixing [30].

An optimal tool to perform such analysis is that of Effective Field Theory (EFT), which allows us to test the SM in a model-independent way. In fact, in addition to studies within specific NP scenarios [1, 2, 31-37], the EFT language has been introduced [1, 2, 38]. However, an EFT approach has not been used yet for global studies of the $s \rightarrow u$ transitions beyond the $\mathrm{U}(3)^{5}$-symmetric limit ${ }^{1}$ where the only NP probe is the CKM unitarity test, given by precise determinations of $\left|V_{u s}\right|$ and $\left|V_{u d}\right|$ [38]. Notice the difference with the $d \rightarrow u$ decays, where global EFT fits have been performed by various groups [39-41].

In this paper we amend these limitations, giving the natural next step in the analysis of (semi)leptonic kaon decays:

- We do not assume any flavor symmetry, generalizing in this way the phenomenological EFT analysis performed in the $\mathrm{U}(3)^{5}$-symmetric limit in ref. [38].

- We keep all operators at the same time. Notice that non-trivial correlations are possible, not only between NP Wilson coefficients (WC) but also involving QCD parameters that are extracted phenomenologically. This generalizes previous works [1, 2, 32], which are covered by our study as specific cases, as we will explicitly show.

- We investigate the complementarity with nuclear, neutron and hyperon $\beta$ decays, which are driven by the same underlying $D \rightarrow u \ell \nu$ transition $(D=s, d)$.

- We provide numerical bounds for the WC. They are to be confirmed by the experimental collaborations taking into account certain correlations not publicly available.

- We match with the so-called SMEFT, i.e. the EFT of the SM at the electroweak (EW) scale, with a linear realization of the electroweak symmetry breaking [42]. This makes possible to study the interplay with high-energy searches, as it was done in ref. [38] in the limit of the flavor symmetry $\mathrm{U}(3)^{5}$. Notice that these flavor-physics studies are fairly clean probes of a small number of WC (compared with searches in colliders). Thus, an interesting degree of complementarity is expected.

Let us stress that our analysis includes the SM limit as a specific case. In fact our output are not only the bounds on the various WC, but also the $V_{u s}$ and $V_{u d}$ CKM matrix elements, and includes various QCD form factors parameters. In the SM limit we recover

\footnotetext{
${ }^{1} \mathrm{U}(3)^{5}$ refers to the flavor symmetry of the SM gauge Lagrangian, i.e. the freedom to perform $\mathrm{U}(3)$ rotations in family space for each of the five fermionic gauge multiplets: $Q_{L}=\left(u_{L}, d_{L}\right), u_{R}, d_{R}, L_{L}=$ $\left(\nu_{L}, e_{L}\right), e_{R}$
} 
the most precise of them [2,43], with small improvements due to the inclusion of the individual rate of $K_{\mu 2}$ as a separate input and the Callan-Treiman theorem.

The outline of the paper is the following. In section 2 we briefly introduce the EFT framework, which we use in section 3 to analyze the channels $K \rightarrow \pi \ell \nu, P \rightarrow \ell \nu(P=$ $K, \pi)$ and baryon $\beta$ decays. This section contains all the relevant formulas, expressing our observables in terms of the parameters of our fit. Section 4 describes how to analyze experimental data with all WC present simultaneously. Section 5 describe the numerical aspects of our analysis and the results of our fit. Then, section 6 contains the running of our bounds to the EW scale, the translation to the SMEFT WC and a brief comparison with LHC searches. Finally, in section 7 we conclude.

\section{The low-energy effective Lagrangian}

The low-scale $O(1 \mathrm{GeV})$ effective Lagrangian for $D \rightarrow u$ transitions $(D=s, d)$ is [38]:

$$
\begin{aligned}
\mathcal{L}_{\mathrm{eff}}=-\frac{G_{F}^{(0)} V_{u D}}{\sqrt{2}} & \sum_{\ell=e, \mu}\left[\left(1+\epsilon_{L}^{D \ell}\right) \bar{\ell} \gamma_{\mu}\left(1-\gamma_{5}\right) \nu_{\ell} \cdot \bar{u} \gamma^{\mu}\left(1-\gamma_{5}\right) D\right. \\
& +\epsilon_{R}^{D \ell} \bar{\ell} \gamma_{\mu}\left(1-\gamma_{5}\right) \nu_{\ell} \bar{u} \gamma^{\mu}\left(1+\gamma_{5}\right) D+\bar{\ell}\left(1-\gamma_{5}\right) \nu_{\ell} \cdot \bar{u}\left[\epsilon_{S}^{D \ell}-\epsilon_{P}^{D \ell} \gamma_{5}\right] D \\
& \left.+\epsilon_{T}^{D \ell} \bar{\ell} \sigma_{\mu \nu}\left(1-\gamma_{5}\right) \nu_{\ell} \cdot \bar{u} \sigma^{\mu \nu}\left(1-\gamma_{5}\right) D\right]+ \text { h.c. },
\end{aligned}
$$

where we use $\sigma^{\mu \nu}=i\left[\gamma^{\mu}, \gamma^{\nu}\right] / 2$ and $G_{F}^{(0)} \equiv \sqrt{2} g^{2} /\left(8 M_{W}^{2}\right)$ is the tree-level definition of the Fermi constant. The latter is obtained from muon decay, which can be also affected by NP effects entering through the normalization of the $\mu \rightarrow e \nu_{\mu} \bar{\nu}_{e}$ effective vertex [38]:

$$
G_{\mu}=G_{F}^{(0)}\left(1+\frac{\delta G_{F}}{G_{F}}\right)
$$

In the derivation of the effective Lagrangian in eq. (2.1) we have assumed that potential right-handed neutrino fields (sterile with respect to the SM gauge group) are heavy compared to the low-energy scale. ${ }^{2}$ We focus on $C P$-even observables, and therefore only the real parts of the WC will interfere with the SM. For the sake of brevity we will write simply $\epsilon_{i}$ instead of $\operatorname{Re}\left(\epsilon_{\mathrm{i}}\right)$ hereafter.

The $\epsilon_{i}^{D \ell}$ coefficients carry a $\sim v^{2} / \Lambda^{2}$ dependence on the NP scale $\Lambda$ and in the SM they vanish leaving the $V-A$ structure generated by the exchange of a $W$ boson. If the NP is coming from dynamics at $\Lambda \gg v$ and electro-weak symmetry breaking is linearly realized, then one can use an $\mathrm{SU}(2)_{L} \times \mathrm{U}(1)_{Y}$ invariant effective theory $[38,42,44,45]$. In this case $[32,38,46]$ :

$$
\epsilon_{R}^{D e}=\epsilon_{R}^{D \mu}+\mathcal{O}\left(v^{4} / \Lambda^{4}\right) \equiv \epsilon_{R}^{D},
$$

so that, up to a subleading corrections in the EFT expansion, a NP effect involving a righthanded current necessarily involves a Higgs-current fermion-current operator [42] and its contribution must be lepton universal.

\footnotetext{
${ }^{2}$ Let us notice that the inclusion of operators with right-handed neutrinos is not expected to affect our results, as they do not interfere with the SM amplitude and thus contribute at $\mathcal{O}\left(\epsilon_{i}^{2}\right)$ to the observables.
} 
Taking into account the points above, and working to linear order in the NP couplings, we can re-express the Lagrangian (2.1) as [47]:

$$
\begin{aligned}
\mathcal{L}_{\mathrm{eff}}= & -\frac{G_{F} \tilde{V}_{u D}^{\ell}}{\sqrt{2}}\left[\bar{\ell} \gamma_{\mu}\left(1-\gamma_{5}\right) \nu_{\ell} \cdot \bar{u}\left[\gamma^{\mu}-\left(1-2 \epsilon_{R}^{D}\right) \gamma^{\mu} \gamma_{5}\right] D\right. \\
& \left.+\bar{\ell}\left(1-\gamma_{5}\right) \nu_{\ell} \cdot \bar{u}\left[\epsilon_{S}^{D \ell}-\epsilon_{P}^{D \ell} \gamma_{5}\right] D+\epsilon_{T}^{D \ell} \bar{\ell} \sigma_{\mu \nu}\left(1-\gamma_{5}\right) \nu_{\ell} \cdot \bar{u} \sigma^{\mu \nu}\left(1-\gamma_{5}\right) D\right] \\
& +\mathcal{O}\left(\epsilon^{2}\right)+\text { h.c. }
\end{aligned}
$$

where

$$
\tilde{V}_{u D}^{\ell}=\left(1+\epsilon_{L}^{D \ell}+\epsilon_{R}^{D}-\frac{\delta G_{F}}{G_{F}}\right) V_{u D}
$$

In addition to $\tilde{V}_{u d}^{e}$ and $\tilde{V}_{u s}^{e}$, we have a total of 16 (combinations of) WC describing the NP modifications to the charged-current decays $D \rightarrow u \ell \nu$ in the SM. The form of the Lagrangian is convenient as it allows to separate the effects of a combination of currentcurrent operators affecting the normalization of the rates and which can be only accessed through CKM-unitarity and lepton-universality tests.

\subsection{Renormalization and scale running of the Wilson coefficients}

The WC display renormalization-scale dependence that is to be canceled in the observables by the opposite dependence in the quantum corrections to the matrix elements of the decays. For instance in QCD we have, at one loop:

$$
\epsilon_{i}^{D \ell}(\mu)=\left(\frac{\alpha_{s}(\mu)}{\alpha_{s}(\Lambda)}\right)^{-\frac{\gamma_{i}}{\beta_{0}}} \epsilon_{i}^{D \ell}(\Lambda),
$$

where $\alpha_{s}$ is the strong structure constant, $\beta_{0}=11-2 / 3 N_{f}$ is the one-loop QCD $\beta$-function coefficient for $N_{f}$ dynamical quark flavors, and $\gamma_{L, R}=0, \gamma_{S, P}=-4$ and $\gamma_{T}=4 / 3$ are the one-loop coefficients of the corresponding anomalous dimensions. ${ }^{3}$

One can also consider the renormalization of the effective operators with respect to electroweak corrections. Although they are very small, they are important for the accuracy of the SM predictions [48, 49], and they induce mixing among certain NP operators that can have interesting phenomenological consequences [50-53]. In our case it is important to take into account the mixing they induce between the (pseudo)scalar and tensor operators, since $\pi_{\ell 2}$ and $K_{\ell 2}$ set very strong bounds on the pseudoscalar couplings. Expressing the results obtained in ref. [52] in terms of the $\mathrm{WC} \vec{\epsilon}(\mu)=\left(\epsilon_{S}^{D \ell}(\mu), \epsilon_{P}^{D \ell}(\mu), \epsilon_{T}^{D \ell}(\mu)\right)$ we obtain:

$$
\frac{d \vec{\epsilon}(\mu)}{d \log \mu}=\frac{\alpha}{2 \pi} \gamma_{\mathrm{ew}} \vec{\epsilon}(\mu)
$$

where

$$
\gamma_{\mathrm{ew}}=\left(\begin{array}{ccc}
-\frac{3}{2 s_{w}^{2}}-\frac{113}{36 c_{w}^{2}} & -\frac{5}{12 c_{w}^{2}} & 3\left(\frac{3}{s_{w}^{2}}+\frac{5}{c_{w}^{2}}\right) \\
-\frac{5}{12 c_{w}^{2}} & -\frac{3}{2 s_{w}^{2}}-\frac{113}{36 c_{w}^{2}} & 3\left(\frac{3}{s_{w}^{2}}+\frac{5}{c_{w}^{2}}\right) \\
\frac{1}{32}\left(\frac{3}{s_{w}^{2}}+\frac{5}{c_{w}^{2}}\right) & \frac{1}{32}\left(\frac{3}{s_{w}^{2}}+\frac{5}{c_{w}^{2}}\right) & -\frac{3}{s_{w}^{2}}-\frac{103}{36 c_{w}^{2}}
\end{array}\right)
$$

where $\alpha$ is the electromagnetic structure constant, $s_{w}^{2}=\sin ^{2} \theta_{W}$ and $c_{w}^{2}=\cos ^{2} \theta_{W}$.

\footnotetext{
${ }^{3}$ In our conventions, at one loop we have $\frac{d \epsilon_{i}}{d \log \mu}=\gamma_{i} \frac{\alpha_{s}}{2 \pi} \epsilon_{i}$ and $\frac{d \alpha_{s}}{d \log \mu}=-\beta_{0} \frac{\alpha_{s}^{2}}{2 \pi}$.
} 


\section{Decay observables}

In this section we calculate the various observables relevant for our analysis in terms of the low-energy WC $\epsilon_{i}$. All the calculations are performed at tree level and the only loop effect taken into account will be the (log-enhanced) running of the Wilson Coefficients described in the previous section.

Nonetheless, it is interesting to notice that our expressions are actually valid at any loop order if the $\epsilon_{i}$ couplings were defined at the amplitude level for each channel (see ref. [54] for a similar description of Higgs decays). The matching to the low-energy EFT can then later performed at any desired order. Since here we match at tree level we do not make this distinction.

\section{$3.1 \quad \pi_{\ell 2(\gamma)}$ and $K_{\ell 2(\gamma)}$}

The photon-inclusive ${ }^{4}$ decay rates are given by

$$
\Gamma_{P_{\ell 2}(\gamma)}=\frac{G_{F}^{2}\left|\tilde{V}_{u D}^{\ell}\right|^{2} f_{P^{ \pm}}^{2}}{8 \pi} m_{P^{ \pm}} m_{\ell}^{2}\left(1-\frac{m_{\ell}^{2}}{m_{P^{ \pm}}^{2}}\right)^{2}\left(1+\delta_{\mathrm{em}}^{P \ell}\right)\left(1+\Delta_{\ell 2}^{P}\right),
$$

where $D=d, s$ for $P=\pi, K$ respectively, $f_{P^{ \pm}}$is the QCD semileptonic decay constant of $P^{ \pm}, \delta_{\mathrm{em}}^{P \ell}$ is the corresponding electromagnetic correction and $\Delta_{\ell 2}^{P}$ contains the NP correction not absorbed in $\tilde{V}_{u D}^{\ell}$.

The electromagnetic corrections are given by $[16,17,49,55]$

$$
1+\delta_{\mathrm{em}}^{P \ell}=S_{\mathrm{ew}}\left\{1+\frac{\alpha}{\pi}\left[F\left(m_{\ell}^{2} / m_{P}^{2}\right)+\frac{3}{2} \log \frac{m_{P}}{m_{\rho}}-c_{1}^{P}\right]\right\}+\mathcal{O}\left(e^{2} p^{4}\right)
$$

where $\alpha$ is the structure constant, $S_{\text {ew }}=1.0232(3)$ [49] encodes universal short distance corrections to the semileptonic transitions in the SM at $\mu=m_{\rho}$ and $F(z)$ describes the leading universal long-distance radiative corrections to a point-like meson [48, 49]. The constant $c_{1}^{P}$ encodes hadronic structure effects that can be calculated in Chiral Perturbation Theory [15-17]. These corrections are at the $1-3 \%$ level with an uncertainty quite smaller than the current one of $f_{P}$.

The NP contribution enters at tree level from the Lagrangian in eq. (2.4):

$$
\begin{aligned}
\Delta_{\ell 2}^{P} & =\left|1-2 \epsilon_{R}^{D}-\frac{m_{P^{ \pm}}^{2}}{m_{\ell}\left(m_{D}+m_{u}\right)} \epsilon_{P}^{D \ell}\right|^{2}-1 \\
& =-4 \epsilon_{R}^{D}-\frac{2 m_{P^{ \pm}}^{2}}{m_{\ell}\left(m_{D}+m_{u}\right)} \epsilon_{P}^{D \ell}+\mathcal{O}\left(v^{4} / \Lambda^{4}\right),
\end{aligned}
$$

where in the second line we have linearized in the WC by expanding up to leading order in the EFT expansion. A very important feature of $P_{\ell 2}$ is its high sensitivity to pseudoscalar contributions because they lift the chiral suppression of the SM. This appears in eqs. (3.1), (3.3) in the coefficients of $\epsilon_{P}^{D \ell}$ which are multiplied by the inverse of the lepton masses. Besides that, partial conservation of the axial current (PCAC) implies the

\footnotetext{
${ }^{4}$ Depending on the channel it might be more convenient (experimentally) to define this rate as fully photon-inclusive $(P=\pi$ case) or to include only the "internal-bremsstrahlung" contribution $(P=K$ case) .
} 
appearance of the quark masses also in the denominators, although always in combination with the meson masses squared in the numerator. Note that the latter combination can be re-expressed using the Gell-Mann-Renner-Oakes equations as $m_{P}^{2} /\left(m_{u}+m_{D}\right) \simeq B_{0}$, which is a nonperturbative parameter that is related to the quark condensate and the pion semileptonic decay constant, $B_{0}(\mu=2 \mathrm{GeV})=-2\langle\bar{u} u\rangle / f_{\pi}^{2} \simeq 2.5 \mathrm{GeV}$. Hence, the contributions from the pseudoscalar operators to the electronic (muonic) mode are enhanced by a factor $\sim 5000(\sim 25)$ with respect to the SM. This means that the linearization performed in eq. (3.3) is only valid for very small values of the WC $\epsilon_{i}$. We will assume this is the case in the initial numerical analysis, and, afterwards, we will discuss how the limits are relaxed once we take into account the very narrow region in the parameter space where quadratic corrections dominate.

The theoretical uncertainty in the SM prediction of these decays can be minimized by taking convenient ratios among the four possible (CP-averaged) channels. The leptonuniversality ratios $R_{P}=\Gamma\left(P_{e 2(\gamma)}\right) / \Gamma\left(P_{\mu 2(\gamma)}\right)$ have been very accurately predicted in the SM because the decay constants $f_{P^{ \pm}}$cancel exactly in the ratio and the radiative corrections are known up to order $\mathcal{O}\left(e^{2} p^{4}\right)$ because the constant $c_{1}^{P}$ disappears from $\delta_{\mathrm{em}}^{P e}-\delta_{\mathrm{em}}^{P \mu}[16,17]$. The dependence on the NP coefficients of $R_{P}$ is:

$$
\begin{aligned}
\frac{R_{P}}{\left.R_{P}\right|_{\mathrm{SM}}} & \equiv \frac{\left|\tilde{V}_{u D}^{e}\right|^{2}}{\left|\tilde{V}_{u D}^{\mu}\right|^{2}}\left(1+\Delta_{e 2 / \mu 2}^{P}\right) \\
& =\frac{\left|\tilde{V}_{u D}^{e}\right|^{2}}{\left|\tilde{V}_{u D}^{\mu}\right|^{2}}\left(1-2 B_{0}\left(\frac{\epsilon_{P}^{D e}}{m_{e}}-\frac{\epsilon_{P}^{D \mu}}{m_{\mu}}\right)\right)+\mathcal{O}\left(v^{4} / \Lambda^{4}\right) \\
& =1+2\left(\epsilon_{L}^{D e}-\epsilon_{L}^{D \mu}\right)-2 B_{0}\left(\frac{\epsilon_{P}^{D e}}{m_{e}}-\frac{\epsilon_{P}^{D \mu}}{m_{\mu}}\right)+\mathcal{O}\left(v^{4} / \Lambda^{4}\right) .
\end{aligned}
$$

Note that the dependence on NP right-handed currents completely disappears at this order as a consequence of eq. (2.3). It is convenient to define $\Delta_{L}^{D}=\epsilon_{L}^{D \mu}-\epsilon_{L}^{D e}$, as this combination of WC will appear several times in our analysis.

The ratio $R_{\ell}=\Gamma\left(K_{\ell 2(\gamma)}\right) / \Gamma\left(\pi_{\ell 2(\gamma)}\right)$ is interesting because $f_{K} / f_{\pi}$ [5] is calculated in the lattice more accurately than the decay constants separately and the combination of radiative corrections entering is independent of the low-energy constants in the hadronstructure functions $c_{1}^{P}$ at $\mathcal{O}\left(e^{2} p^{2}\right)[21]$. From this ratio one can obtain

$$
\begin{aligned}
R_{\ell} & \rightarrow \frac{\left|\tilde{V}_{u s}^{\ell}\right|^{2} f_{K^{ \pm}}^{2}}{\left|\tilde{V}_{u d}^{\ell}\right|^{2} f_{\pi^{ \pm}}^{2}}\left(1+\Delta_{\ell 2}^{K / \pi}\right) \\
& =\frac{\left|\tilde{V}_{u s}^{\ell}\right|^{2} f_{K^{ \pm}}^{2}}{\left|\tilde{V}_{u d}^{\ell}\right|^{2} f_{\pi^{ \pm}}^{2}}\left(1-4\left(\epsilon_{R}^{s}-\epsilon_{R}^{d}\right)-\frac{2 B_{0}}{m_{\ell}}\left(\epsilon_{P}^{s \ell}-\epsilon_{P}^{d \ell}\right)\right)+\mathcal{O}\left(v^{4} / \Lambda^{4}\right)
\end{aligned}
$$

Notice that $R_{\ell}$ is not only sensitive to the NP-modified CKM elements $\left|\tilde{V}_{u s}^{\ell}\right| /\left|\tilde{V}_{u d}^{\ell}\right|$ but also, and independently, to the right-handed or pseudoscalar operators. Last, we note that this result is in agreement with ref. [1] (eq. 2.37). ${ }^{5}$

\footnotetext{
${ }^{5}$ The relation between their $\mathrm{WC}$ and ours is the following: $c_{L L}^{V}=-1-\epsilon_{L}^{*}, c_{R L}^{V}=-\epsilon_{R}^{*}, c_{R R}^{S}=-\left(\epsilon_{S}^{*}+\right.$ $\left.\epsilon_{P}^{*}\right) / 2, c_{L R}^{S}=-\left(\epsilon_{S}^{*}-\epsilon_{P}^{*}\right) / 2$ and $c_{R R}^{T}=-\epsilon_{T}^{*}$ (flavor indexes implicit). The remaining coefficients are zero in our EFT since they involve operators with right-handed neutrinos.
} 


\section{$3.2 \quad P_{\ell 2 \gamma}$}

For the sake of completeness we discuss now briefly radiative pion and kaon decays, $P \rightarrow$ $\ell \nu \gamma\left(P_{\ell 2 \gamma}\right)$, as NP probes. In addition to the QED correction (internal-bremsstrahlung) to the $P \rightarrow \ell \nu$ decay, we have the so-called "structure-dependent" terms which can be extracted separately from experiment [56-58]; in the SM they depend on $P \rightarrow \gamma$ hadronic form factors and some of them are not chirally suppressed $[3,59]$. The interest of $P_{\ell 2 \gamma}$ in the context of NP is that their kinematic distributions are sensitive to the tensor operator $[60$, 61], which enter with new form factors [62] $(q=p-k)$ :

$$
\begin{aligned}
\left\langle\gamma(k, \epsilon)\left|\bar{u} \sigma^{\mu \nu} \gamma_{5} D\right| P^{-}(p)\right\rangle= & \frac{e}{2} F_{T}^{P}\left(q^{2}\right)\left(\epsilon^{\mu} k^{\nu}-\epsilon^{\nu} k^{\mu}\right) \\
& +G_{T}^{P}\left(q^{2}\right)\left[\epsilon \cdot p\left(p^{\mu} k^{\nu}-p^{\nu} k^{\mu}\right)+q \cdot p\left(\epsilon^{\mu} p^{\nu}-\epsilon^{\nu} p^{\mu}\right)\right],
\end{aligned}
$$

although $G_{T}^{P}$ is kinematically suppressed in the amplitude and can be neglected in first approximation. In fact, the PIBETA collaboration has obtained a stringent constraint on these contributions in $\pi_{e 2 \gamma}$ [57] that, using the calculation of $F_{T}^{\pi}$ obtained in ref. [62], leads to the bound [47]:

$$
-1.2 \times 10^{-3} \leq \epsilon_{T}^{d e} \leq 1.36 \times 10^{-3} \quad(90 \% \text { C.L. }) .
$$

Similar experimental analyses have not been performed with $\pi_{\mu 2 \gamma}$ or $K_{\ell 2 \gamma}$ yet, where the experimental precision is not so high. Calculations of the kaon tensor form factors are also lacking, while the muonic channels are expected to be less sensitive to tensor interactions, as they are dominated by the internal-bremsstrahlung part.

Finally, the structure-dependent terms also depend on the vector and axial $D \rightarrow u \ell \nu$ currents (e.g. the SM) and, therefore, on $\left|\tilde{V}_{u D}^{\ell}\right|$ and $\epsilon_{R}^{D}$. However, in order to provide competitive values for these quantities one would need high-accuracy data and LQCD results for the corresponding SM form factors.

\section{$3.3 \quad K_{\ell 3(\gamma)}$}

In the SM, the $K \rightarrow \pi \ell \nu$ decay amplitude depends on the hadronic matrix element [1]:

$$
\left\langle\pi^{-}(k)\left|\bar{s} \gamma^{\mu} u\right| K^{0}(p)\right\rangle=P^{\mu} f_{+}\left(q^{2}\right)+q^{\mu} f_{-}\left(q^{2}\right),
$$

where the $K^{0} \pi^{-}$channel is taken as reference, $P=p+k$ and $q=p-k$. The $f_{-}\left(q^{2}\right)$ can be written in terms of $f_{+}\left(q^{2}\right)$ and the scalar form factor $f_{0}\left(q^{2}\right)$ using the conservation of the vector current in $\mathrm{QCD}$,

$$
\begin{aligned}
\left\langle\pi^{-}(k)|\bar{s} u| K^{0}(p)\right\rangle & =-\frac{m_{K^{0}}^{2}-m_{\pi^{ \pm}}^{2}}{m_{s}-m_{u}} f_{0}\left(q^{2}\right), \\
f_{0}\left(q^{2}\right) & =f_{+}\left(q^{2}\right)+\frac{q^{2}}{m_{K^{0}}^{2}-m_{\pi^{ \pm}}^{2}} f_{-}\left(q^{2}\right),
\end{aligned}
$$

Finally, in presence of a tensor operator a new form factor appears [1]:

$$
\left\langle\pi^{-}(k)\left|\bar{s} \sigma^{\mu \nu} u\right| K^{0}(p)\right\rangle=i \frac{p^{\mu} k^{\nu}-k^{\mu} p^{\nu}}{m_{K^{0}}} B_{T}\left(q^{2}\right) .
$$




\subsubsection{Kinematical distribution}

First let us briefly review the situation in the SM $[1,2]$. There are various methods proposed for the parametrization of the $q^{2}$ dependence of the form factors. The conventional one relies on a Taylor expansion,

$$
\bar{f}_{+, 0}\left(q^{2}\right)=\frac{f_{+, 0}\left(q^{2}\right)}{f_{+, 0}(0)}=1+\lambda_{+, 0}^{\prime} \frac{q^{2}}{m_{\pi}^{2}}+\frac{1}{2} \lambda_{+, 0}^{\prime \prime}\left(\frac{q^{2}}{m_{\pi}^{2}}\right)^{2}+\ldots
$$

where the higher orders terms are negligible in the kinematic range of the decay, $q^{2} \in$ $\left[m_{\ell}^{2},\left(m_{K}-m_{\pi}\right)^{2}\right]$. These parameters are customarily fitted to the kinematic distributions of the decay (or Dalitz plot) [63-70], allowing for a calculation the phase-space integral (see next subsection).

The Taylor-expansion parametrization introduces a number of parameters which can not be always determined experimentally free of ambiguities and more efficient parametrizations have been proposed [1, 11, 20,32], incorporating physical constraints to reduce the number of independent parameters. In particular, for $f_{0}\left(q^{2}\right)$ one can use a dispersive representation $[11,20,32]$ that allows one to relate all its slope parameters to a single quantity that needs to be measured and that is chosen to be $\log C$, where

$$
C=\bar{f}_{0}\left(m_{K}^{2}-m_{\pi}^{2}\right) .
$$

Importantly, the value of this quantity can be determined very precisely in QCD using the Callan-Treiman theorem (CTT) [71]

$$
C_{\mathrm{QCD}}=\frac{f_{K}}{f_{\pi}} \frac{1}{f_{+}(0)}+\Delta_{\mathrm{CT}}
$$

where $\Delta_{\mathrm{CT}}=-0.0035(80)$ is a small $\mathcal{O}\left(m_{u, d} /\left(4 \pi f_{\pi}\right)\right)$ correction calculated using ChPT $[8,72]$.

It is also interesting to note that LQCD calculations of the $q^{2}$-dependence of the SM form factors have recently appeared [73], although their precision is still smaller than the experimental determinations. Their inclusion in the future should be straightforward and it should help obtaining a more precise $\left|\tilde{V}_{u s}^{\ell}\right|$ determination and stronger NP bounds, while at the same time making the SM calculations more robust.

Scalar and tensor operators modify the kinematic distribution and they should be determined together with the form factor parameters in the fits to the Dalitz plots. First of all, their interference with the SM is proportional to the lepton mass due to their chiralityflipping nature. A consequence of this is that the dependence on the corresponding WC for the electronic mode is, in very good approximation, quadratic and their kinematic distributions are SM-like at leading order of the EFT expansion.

In the muon channel, the effect of the scalar operator can be absorbed in the scalar form factor [1]. This can be easily seen at the very amplitude level:

$$
\begin{aligned}
\mathcal{M}\left(K^{0} \rightarrow \pi^{-} \mu \nu\right)= & -\frac{G_{F} \tilde{V}_{u s}^{\mu *}}{\sqrt{2}}\left[\left(\bar{u} \not P\left(1-\gamma_{5}\right) v+m_{\mu} \frac{m_{K^{0}}^{2}-m_{\pi^{ \pm}}^{2}}{q^{2}} \bar{u}\left(1+\gamma_{5}\right) v\right) f_{+}\left(q^{2}\right)\right. \\
& \left.-m_{\mu} \frac{m_{K^{0}}^{2}-m_{\pi^{ \pm}}^{2}}{q^{2}} f_{0}\left(q^{2}\right)\left(1+\epsilon_{S}^{s \mu} \frac{q^{2}}{m_{\mu}\left(m_{s}-m_{u}\right)}\right) \bar{u}\left(1+\gamma_{5}\right) v+\ldots\right],
\end{aligned}
$$


where the dots correspond to the tensor contribution that we discuss below. Since this effect vanishes for $q^{2}=0$, it is easy to see that the whole effect of a scalar interaction ends up hidden in the $q^{2}$-dependence of the scalar form factor $f_{0}\left(q^{2}\right)$. If precise values for $f_{+}(0)$ and $f_{K} / f_{\pi}$ are provided in QCD, the CTT gives a very accurate prediction of this form factor at $q^{2}=m_{K}^{2}-m_{\pi}^{2}$, which allows to separate $\epsilon_{S}^{s \mu}$ from the experimental measurement:

$$
\begin{aligned}
\log C_{\text {exp }} & =\log \left[C_{\mathrm{QCD}}\left(1+\frac{m_{K}^{2}-m_{\pi}^{2}}{m_{\mu}\left(m_{s}-m_{u}\right)} \epsilon_{S}^{s \mu}\right)\right] \\
& =\log C_{\mathrm{QCD}}+\frac{m_{K}^{2}-m_{\pi}^{2}}{m_{\mu}\left(m_{s}-m_{u}\right)} \epsilon_{S}^{s \mu}+\mathcal{O}\left(v^{4} / \Lambda^{4}\right) .
\end{aligned}
$$

On the other hand, the tensor term can not be described by a simple re-definition of the SM contributions. This can be appreciated better by looking at the differential decay rate in terms $q^{2}$ and the angle $\theta$ defined in the $q$ rest frame by the 3 -momenta of the charged lepton and the one of the recoiling pion, $\vec{k}$ :

$$
\begin{aligned}
\frac{d \Gamma}{d q^{2} d(\cos \theta)}= & \frac{G_{F}^{2}\left|\tilde{V}_{u s}^{\mu}\right|^{2}}{128 \pi^{3}} C_{K} S_{\mathrm{ew}}\left(1+\delta^{c}+\delta_{\mathrm{em}}^{c \mu}\left(q^{2}, \theta\right)\right)^{2} \frac{|\vec{k}|}{m_{K}^{2}}\left(1-\frac{m_{\mu}^{2}}{q^{2}}\right)^{2} \\
& \times\left\{\sin ^{2} \theta\left|2 m_{K}\right| \vec{k}\left|f_{+}\left(q^{2}\right)\left(1-\frac{2 \epsilon_{T}^{s \mu} m_{\mu}}{m_{K}} \frac{B_{T}\left(q^{2}\right)}{f_{+}\left(q^{2}\right)}\right)\right|^{2}\right. \\
& \left.+m_{\mu}^{2}\left|\frac{2 m_{K}|\vec{k}|}{\sqrt{q^{2}}} \cos \theta f_{+}\left(q^{2}\right)\left(1-\frac{2 \epsilon_{T}^{s \mu} q^{2}}{m_{\mu} m_{K}} \frac{B_{T}\left(q^{2}\right)}{f_{+}\left(q^{2}\right)}\right)+\frac{m_{K}^{2}-m_{\pi}^{2}}{\sqrt{q^{2}}} \tilde{f}_{0}\left(q^{2}\right)\right|^{2}\right\},
\end{aligned}
$$

where $C_{K}=1(1 / 2)$ for the neutral (charged) kaons, $\tilde{f}_{0}\left(q^{2}\right)=f_{0}\left(q^{2}\right)\left(1+\epsilon_{S}^{s \mu} \frac{q^{2}}{m_{\mu}\left(m_{s}-m_{u}\right)}\right)$ denotes the scalar form factor modified by NP, $\delta_{\mathrm{em}}^{c \mu}\left(q^{2}, \theta\right)$ are radiative corrections and $\delta^{c}$ is the isospin-breaking correction for the charged kaon channel, which can be obtained in ChPT $[9,12,15,18,19]$. It is evident that the tensor operator introduces a characteristic dependence on $q^{2}$ and $\theta$ that is different from the SM.

\subsubsection{Total rates}

The photon-inclusive $K_{\ell 3}$ total decay rates can be written as [1]

$$
\Gamma\left(K_{\ell 3(\gamma)}\right)=\frac{G_{F}^{2} m_{K}^{5}}{192 \pi^{3}} C_{K} S_{\mathrm{ew}}\left|\tilde{V}_{u s}^{\ell}\right|^{2} f_{+}(0)^{2} I_{K}^{\ell}\left(\lambda_{+, 0}, \epsilon_{S, T}^{s \ell}\right)\left(1+\delta^{c}+\bar{\delta}_{\mathrm{em}}^{c \ell}\right)^{2},
$$

where $\bar{\delta}_{\mathrm{em}}^{c \ell}$ is the integrated radiative correction and $I_{K}^{\ell}\left(\lambda_{+, 0}, \epsilon_{S, T}^{s \ell}\right)$ is the phase space integral, where $\lambda_{+, 0}$ should be interpreted as a generic reference to the parameters describing 
the $q^{2}$ dependence of the form factors. Its expression is given by

$$
\begin{aligned}
I_{K}^{\ell}= & I_{K, 0}^{\ell}-\epsilon_{T}^{s \ell} I_{T}^{\ell}+\mathcal{O}\left(v^{4} / \Lambda^{4}\right) \\
I_{K, 0}^{\ell}= & \frac{1}{f_{+}(0)^{2}} \int \frac{d q^{2}}{m_{K}^{2}} \lambda^{3 / 2}\left(q^{2}\right)\left(1+\frac{m_{\ell}^{2}}{2 q^{2}}\right)\left(1-\frac{m_{\ell}^{2}}{q^{2}}\right)^{2} \\
& \times\left(f_{+}\left(q^{2}\right)^{2}+\frac{3 m_{\ell}^{2}\left(m_{K}^{2}-m_{\pi}^{2}\right)^{2}}{\left(2 q^{2}+m_{\ell}^{2}\right) m_{K}^{4} \lambda\left(q^{2}\right)} \tilde{f}_{0}\left(q^{2}\right)^{2}\right), \\
I_{T}^{\ell}= & \frac{1}{f_{+}(0)^{2}} \int \frac{d q^{2}}{m_{K}^{2}} \lambda^{3 / 2}\left(q^{2}\right) \frac{6 m_{\ell}}{m_{K}}\left(1-\frac{m_{\ell}^{2}}{q^{2}}\right)^{2} B_{T}\left(q^{2}\right) f_{+}\left(q^{2}\right),
\end{aligned}
$$

where $\lambda\left(q^{2}\right)=1-2 r_{\pi}+r_{\pi}^{2}-2 q^{2} / m_{K}^{2}-2 r_{\pi} q^{2} / m_{K}^{2}+q^{4} / m_{K}^{4}$ and $r_{\pi}=m_{\pi}^{2} / m_{K}^{2}$. Let us notice that the tensor contribution to the total rate, $I_{T}^{\ell}$, does not agree with the result shown in the 2008 Flavianet report [1].

To determine the total rates beyond the SM one first needs to perform a global fit of the form-factor parameters and $\epsilon_{T}^{s \ell}$ (provided a value for $B_{T}$ ) to the kinematic distribution in eq. (3.19). This requires a careful assessment of the uncertainty introduced by the radiative corrections $\delta_{\mathrm{em}}^{c l}\left(q^{2}, \theta\right)$ which can introduce sizable corrections to the rates in some regions of the phase space [19]. Besides the parameters corresponding to $f_{+}\left(q^{2}\right)$, for the muonic mode one should obtain from the fit (correlated) intervals for $\log C$ and $\epsilon_{T}^{s \mu}$. As it will be discussed in more detail below, for the electronic mode our framework must be pushed beyond the leading order in the EFT expansion to search for $\left|\epsilon_{S}^{s e}\right|^{2}$ and $\left|\epsilon_{T}^{s e}\right|^{2}$. With the final results of these fits one can now compute the phase-space integral $I_{K}^{\ell}$ in eq. (3.20), which allows for a determination of $f_{+}(0)\left|\tilde{V}_{u s}^{\ell}\right|$ from the total rate $\Gamma\left(K_{\ell 3(\gamma)}\right)$.

Similarly to $P_{\ell 2(\gamma)}$, there is also a lepton-universality ratio in $K_{\ell 3(\gamma)}$ constructed from the total rates in which most of the theoretical uncertainties cancel:

$$
r_{\mu e}=\frac{\Gamma_{K_{\mu 3}} I_{e 3}\left(1+2 \delta_{\mathrm{em}}^{K e}\right)}{\Gamma_{K_{e 3}} I_{\mu 3}\left(1+2 \delta_{\mathrm{em}}^{K \mu}\right)}=\frac{\left|\tilde{V}_{u s}^{\mu}\right|^{2}}{\left|\tilde{V}_{u s}^{e}\right|^{2}}=1+2 \Delta_{L}^{s}+\mathcal{O}\left(v^{4} / \Lambda^{4}\right),
$$

and that is only sensitive to the difference of left-handed $s \rightarrow u$ currents, $\Delta_{L}^{s}=\epsilon_{L}^{s \mu}-\epsilon_{L}^{s e}$, up to $\mathcal{O}\left(v^{2} / \Lambda^{2}\right)$ due to eq. (2.3).

\subsection{Nuclear, neutron and hyperon $\beta$ decay}

The semileptonic decays of nuclei, neutron and hyperons are mediated by the same effective Lagrangian as the (semi)leptonic pion and kaons decays. We summarize here the aspects of these decays that offer the strongest synergies.

The most accurate value for $\left|\tilde{V}_{u d}^{e}\right|$ is obtained from superallowed nuclear $\beta$ transitions, in an analysis that also sets the most stringent limits on the non-standard scalar Wilson coefficient $\epsilon_{S}^{d e}$ (via the Fierz interference term $b_{F}$ ) [47, 74]. Combining this $\left|\tilde{V}_{u d}^{e}\right|$ determination with the $\left|\tilde{V}_{u s}^{e}\right|$ value obtained from $K_{e 3(\gamma)}$ allows one to test CKM unitarity, which 
probes the following combination of WC [38]:

$$
\begin{aligned}
\left|\tilde{V}_{u d}^{e}\right|^{2}+\left|\tilde{V}_{u s}^{e}\right|^{2} & =1+\Delta_{\mathrm{CKM}}, \\
\Delta_{\mathrm{CKM}} & =2\left|\tilde{V}_{u d}^{e}\right|^{2}\left(\epsilon_{L}^{d e}+\epsilon_{R}^{d}\right)+2\left|\tilde{V}_{u s}^{e}\right|^{2}\left(\epsilon_{L}^{s e}+\epsilon_{R}^{s}\right)-2 \frac{\delta G_{F}}{G_{F}}+\mathcal{O}\left(v^{4} / \Lambda^{4}\right) .
\end{aligned}
$$

where we have neglected the contribution of $\left|\tilde{V}_{u b}^{e}\right|^{2}$ because its value is smaller than the current uncertainty in $\Delta_{\mathrm{CKM}}[4]$.

At the hadron level, neutron and hyperon $\beta$ decays are weighed by different form factors. For the SM contributions we have [75]:

$$
\begin{aligned}
\left\langle B_{2}\left(p_{2}\right)\left|\bar{u} \gamma_{\mu} D\right| B_{1}\left(p_{1}\right)\right\rangle & =\bar{u}_{2}\left(p_{2}\right)\left[f_{1}\left(q^{2}\right) \gamma_{\mu}+\frac{f_{2}\left(q^{2}\right)}{M_{1}} \sigma_{\mu \nu} q^{\nu}+\frac{f_{3}\left(q^{2}\right)}{M_{1}} q_{\mu}\right] u_{1}\left(p_{1}\right), \\
\left\langle B_{2}\left(p_{2}\right)\left|\bar{u} \gamma_{\mu} \gamma_{5} D\right| B_{1}\left(p_{1}\right)\right\rangle & =\bar{u}_{2}\left(p_{2}\right)\left[g_{1}\left(q^{2}\right) \gamma_{\mu}+\frac{g_{2}\left(q^{2}\right)}{M_{1}} \sigma_{\mu \nu} q^{\nu}+\frac{g_{3}\left(q^{2}\right)}{M_{1}} q_{\mu}\right] \gamma_{5} u_{1}\left(p_{1}\right),
\end{aligned}
$$

while the (pseudo)scalar and tensor operators introduce new form factors [47, 75, 76]. The normalization of the decays, $f_{1}(0)\left|\tilde{V}_{u D}^{\ell}\right|$, leads to independent $\left|\tilde{V}_{u D}^{\ell}\right|$ constraints once a theoretical value for the "vector charge" of the transition, $f_{1}(0)$, is used (see e.g. refs. [77-81]).

The only effects of the right-handed currents $\epsilon_{R}^{D}$ in these decays enter hidden in $\left|\tilde{V}_{u D}^{\ell}\right|$, cf. eq. (2.5), and in the axial form factors, like the "axial charge" of the transition, $g_{1} \equiv$ $g_{1}(0)$ (commonly denoted by $g_{A}$ in the case of the neutron decay) $[47,76]$ :

$$
g_{A}^{\text {expt }}=\left(1-2 \epsilon_{R}^{d}\right) g_{A}, \quad g_{1}^{\text {expt }}=\left(1-2 \epsilon_{R}^{s}\right) g_{1} .
$$

Thus, a bound on the right-handed current can be determined if any of the axial form factors is both measured and calculated in LQCD. This is indeed the case for $g_{A}$, which has been measured precisely [82, 83] and for which there are ongoing LQCD efforts [84], with results in the physical point currently at the few-percent level [85-87]. As recently pointed out in ref. [76], our knowledge for the hyperon decays is far less advanced both experimentally and theoretically.

The nonstandard coefficients $\epsilon_{S, P, T}^{D \ell}$ modify not only the total rate but also the kinematic distributions and polarization observables of the $\beta$ decays [47, 76, 88, 89]. Strong bounds on $\epsilon_{S, T}^{d e}$ have been obtained from global fits to various precise measurements in nuclear and neutron decays [39-41, 74, 90], whereas somewhat weaker (but still nontrivial) bounds are expected for the pseudo-scalar term $\epsilon_{P}^{d e}$ [89]. It is also worth noting that the muonic NP-modified CKM matrix element, $\tilde{V}_{u d}^{\mu}$, and WC, $\epsilon_{S, P, T}^{d \mu}$, cannot be determined from $\beta$ decays since the muon channels are kinematically forbidden.

The analysis of these contributions can be extended to semileptonic hyperon decays [76]. Similarly to $K_{\ell 3(\gamma)}$, the chiral suppression of (pseudo)scalar and tensor operators implies that only the muonic case presents a non-negligible linear dependence on the WC. For instance, in ref. [76] the following lepton universality ratio was studied:

$$
R_{B_{1} B_{2}}=\frac{\Gamma\left(B_{1} \rightarrow B_{2} \mu \nu\right)}{\Gamma\left(B_{1} \rightarrow B_{2} e \nu\right)}=\frac{\left|\tilde{V}_{u s}^{\mu}\right|^{2}}{\left|\tilde{V}_{u s}^{e}\right|^{2}}\left(1+R_{S} \epsilon_{S}^{s \mu}+R_{T} \epsilon_{T}^{s \mu}\right)
$$


where the coefficients $R_{S, T}$ depend of the decay channel [76]. The NP contributions to $\left|\tilde{V}_{u s}^{\mu}\right| /\left|\tilde{V}_{u s}^{e}\right|$ are encoded in $\Delta_{L}^{s}$ which can be extracted independently from $K_{\ell 3}$ decays, cf. eq. (3.22), so that measuring $R_{B_{1} B_{2}}$ in different channels allows to set bounds on $\epsilon_{T, S}^{s \mu}$ at the few per-cent level, even though the old hyperon decay data set is used as input [76]. New experiments and a comprehensive analysis of observables is needed to fully exploit the interesting degree of complementarity between hyperon and kaon decays.

\section{Strategy for the global analyses}

Having discussed all the (CP-averaged) observables appearing in $P_{\ell 2(\gamma)}$ and $K_{\ell 3(\gamma)}$, and its complementarity with baryon decays, we will now outline a strategy to take into account all the information about NP one can extract from the experimental data, while summarizing also the theoretical inputs needed.

Only three of the four $P_{\ell 2(\gamma)}$ ratios discussed in section 3.1 are independent and we need also to include in the analysis one total rate (controlling the overall normalization of the rates). We choose $R_{\pi}, R_{K}, R_{\mu}$ and $\Gamma\left(K_{\mu 2(\gamma)}\right)$. For the theoretical predictions we need $f_{K}^{ \pm} / f_{\pi}^{ \pm}$and $f_{K^{ \pm}}$which are calculated accurately in LQCD, and the radiative corrections described in section 3.1. The output quantities obtained are:

$$
\begin{gathered}
\left\{R_{\pi}, R_{K}, R_{\mu}, \Gamma\left(K_{\mu 2(\gamma)}\right)\right\} \\
\downarrow R_{\pi}^{\text {th }}, R_{K}^{\text {th }}, f_{K^{ \pm}} / f_{\pi^{ \pm}}, f_{K^{ \pm}}, \text {radiative corrections } \\
\left\{\frac{\left|\tilde{V}_{u d}^{e}\right|^{2}}{\left|\tilde{V}_{u d}^{\mu}\right|^{2}}\left(1+\Delta_{e 2 / \mu 2}^{\pi}\right), \frac{\left|\tilde{V}_{u s}^{e}\right|^{2}}{\left|\tilde{V}_{u s}^{\mu}\right|^{2}}\left(1+\Delta_{e 2 / \mu 2}^{K}\right), \frac{\left|\tilde{V}_{u s}^{\mu}\right|^{2}}{\left|\tilde{V}_{u d}^{\mu}\right|^{2}}\left(1+\Delta_{\mu 2}^{K / \pi}\right),\left|\tilde{V}_{u s}^{\mu}\right|^{2}\left(1+\Delta_{\mu 2}^{K}\right)\right\}
\end{gathered}
$$

where the $\Delta^{X}$ are the combinations of the WC in eqs. (3.3), (3.4), (3.7).

For $K_{\ell 3(\gamma)}$, we have three (CP-averaged) channels for electron and muon, namely $K^{ \pm}, K_{L}$ and $K_{S}$. Since they are sensitive to the same short-distance physics, we can simply average over them (taking into account SM long-distance effects that affect them differently). The comparison of the output obtained in different channels (e.g. $\left.f_{+}(0)\left|\tilde{V}_{u s}^{\ell}\right|\right)$ is a useful experimental crosscheck $[1,2]$, but it does not provide any NP constraint in the EFT framework.

First, the kinematic distributions have to be fitted to a parametrization of the form factors and, also to $\epsilon_{T}^{s \mu}$ using the LQCD determination for $B_{T}\left(q^{2}\right)$. For the muonic mode, one extracts $\epsilon_{S}^{s \mu}$ comparing the experimental determination of $\log C$ with the value given by the CTT theorem and the lattice calculations of $f_{+}(0)$ and $f_{K} / f_{\pi}$, viz. eqs. (3.16), (3.18).

With the correlated results of these fits one calculates the spectral integrals $I_{K}^{e}$ and $I_{K}^{\mu}$, that are then used to extract $f_{+}(0)\left|\tilde{V}_{u s}^{e}\right|$ and $r_{\mu e}$ from the electronic and muonic total rates, viz. eqs. (3.20), (3.22), which then gives $\left|\tilde{V}_{u s}^{\ell}\right|$ using as input the LQCD determination for 
$f_{+}(0)$. Schematically:

$$
\begin{gathered}
\text { Kinematic distributions } \\
\downarrow \text { radiative corrections } \\
\left\{\lambda_{+}^{\prime}, \lambda_{+}^{\prime \prime}, \log C_{\exp }, B_{T}\left(q^{2}\right) \epsilon_{T}^{s \mu}\right\} \\
\downarrow \log C_{\mathrm{QCD}}, B_{T}\left(q^{2}\right), \\
\left\{r_{\mu e}, \Gamma\left(K_{e 3(\gamma)}\right)\right\} \longrightarrow \\
\left\{I_{K}^{e}, I_{K}^{\mu}, \epsilon_{S}^{s \mu}, \epsilon_{T}^{s \mu}\right\}
\end{gathered}
$$

Note that, in general, and except for $\epsilon_{S, T}^{s \mu}$, the global analysis of $P_{\ell 2(\gamma)}$ and $K_{\ell 3(\gamma)}$ does not allow to determine each WC separately, but only certain combinations of them. Baryon $\beta$ decays provide extra observables that can help to disentangle most of them individually. For instance, including the determination of $\left|\tilde{V}_{u d}^{e}\right|$ allows one to access $\Delta_{\text {CKM via eq. (3.23) }}$ and the WC combination $\Delta_{\mu 2}^{K / \pi}-2 \Delta_{L}^{d}$ from $R_{\mu}$. This leads to a reinterpretation of the classical $\left|V_{u s}\right|-\left|V_{u d}\right|$ plot illustrating the consistency of $K_{\ell 3}, K_{\ell 2} / \pi_{\ell 2}$ and nuclear $\beta$ decays determinations of $\left|V_{u d}\right|$ and $\left|V_{u s}\right|$ [91]. In our EFT approach such test represents a powerful probe of the NP contribution $\Delta_{\mu 2}^{K / \pi}-2 \Delta_{L}^{d}$, whereas the additional consistency with the unitarity condition probes $\Delta_{\text {CKM }}$. We will come back to this point in section 5.2.1.

Last but not least, the analysis of the nucleon and hyperon axial charges allows to extract $\epsilon_{R}^{D}(D=d, s)$, which, in turn, makes possible to set individual bounds on $\epsilon_{P}^{s e}, \epsilon_{P}^{s \mu}$ and $\epsilon_{P}^{d e}$ from the kaon fit output. Schematically:

$$
\begin{gathered}
\left\{\left|\tilde{V}_{u s}^{e}\right|, \Delta_{L}^{s}, \frac{\left|\tilde{V}_{u d}^{e}\right|^{2}}{\left|\tilde{V}_{u d}^{\mu}\right|^{2}}\left(1+\Delta_{e 2 / \mu 2}^{\pi}\right), \frac{\left|\tilde{V}_{u s}^{e}\right|^{2}}{\left|\tilde{V}_{u s}^{\mu}\right|^{2}}\left(1+\Delta_{e 2 / \mu 2}^{K}\right), \frac{\left|\tilde{V}_{u s}^{\mu}\right|^{2}}{\left|\tilde{V}_{u d}^{\mu}\right|^{2}}\left(1+\Delta_{\mu 2}^{K / \pi}\right),\left|\tilde{V}_{u s}^{\mu}\right|^{2}\left(1+\Delta_{\mu 2}^{K}\right)\right\} \\
\left\{\left|\tilde{V}_{u d}^{e}\right|, g_{A}^{\text {expt }}, g_{1}^{\text {expt }}\right\} \longrightarrow g_{A}^{\mathrm{LQCD}}, g_{1}^{\mathrm{LQCD}}, \text { CKM unitarity } \\
\left\{\left|\tilde{V}_{u d}^{e}\right|, \Delta_{\mathrm{CKM}}, \Delta_{L}^{s}, \Delta_{L P}^{d}, \epsilon_{P}^{s e}, \epsilon_{P}^{s \mu}, \epsilon_{P}^{d e}, \epsilon_{R}^{d}, \epsilon_{R}^{s}\right\}
\end{gathered}
$$

There are certain WC which cannot be determined individually using the low-energy data discussed thus far. For instance, the lack of experimental input for a leptonuniversality ratio $\left|\tilde{V}_{u d}^{e}\right|^{2} /\left|\tilde{V}_{u d}^{\mu}\right|^{2}$, precludes setting a bound on the combination $\epsilon_{L}^{d e}-\epsilon_{L}^{d \mu}$ separated from $\epsilon_{P}^{d \mu}$ in the combination:

$$
\Delta_{L P}^{d}=-\Delta_{L}^{d}+\frac{B_{0}}{m_{\mu}} \epsilon_{P}^{d \mu}=\epsilon_{L}^{d e}-\epsilon_{L}^{d \mu}+\frac{B_{0}}{m_{\mu}} \epsilon_{P}^{d \mu},
$$

obtained from $R_{\pi}$. Nonetheless, one gets access to these WC in muon-capture and inverse $\beta$-decay experiments, in which a few-percent experimental precision has been achieved. It 
would be interesting to investigate the potential of these processes to provide independent bounds on NP in the context of the EFT approach described here.

In addition, the $\mathrm{WC}$ in the following combinations:

$$
\begin{aligned}
\tilde{\Delta}_{\mathrm{CKM}} & =2\left|\tilde{V}_{u d}^{e}\right|^{2} \epsilon_{L}^{d e}+2\left|\tilde{V}_{u s}^{e}\right|^{2} \epsilon_{L}^{s e}-2 \frac{\delta G_{F}}{G_{F}} \approx 1.9 \epsilon_{L}^{d e}+0.1 \epsilon_{L}^{s e}-2 \frac{\delta G_{F}}{G_{F}}, \\
\Delta_{L}^{s} & =\epsilon_{L}^{s \mu}-\epsilon_{L}^{s e} .
\end{aligned}
$$

are not determined individually. This can not be improved by adding other low-energy charged-current processes and the only way to access the orthogonal directions to these WC combinations is through the use of high-energy data, or neutral-current low-energy processes connected to those studied in this work due to the $\mathrm{SU}(2)_{L} \times \mathrm{U}(1)_{Y}$ symmetry in the EFT.

In figure 1 we present a flowchart describing the correlation among different low-energy processes in a global (linearized) EFT analysis of NP in $D \rightarrow u \ell \nu$ transitions ( $D=d, s, \ell=$ $e, \mu)$ and summarizing the different experimental and the theoretical inputs that are needed.

\subsection{Quadratic contributions of the WC}

In principle, it is possible to extend our analysis to include quadratic contributions of the WC to the observables, although these count as $\mathcal{O}\left(v^{4} / \Lambda^{4}\right)$ in the EFT expansion and one needs to promote the global analysis to that order. In particular, the relation $\epsilon_{R}^{D e}=\epsilon_{R}^{D \mu}$ would be violated by the interference of the SM with dimension- 8 operators. For the sake of clarity, in this work we restrict ourselves to the few cases where the quadratic contributions can give the leading NP effects.

As discussed in section 3.3, this is the case of the WC $\epsilon_{S, T}^{s e}$, which are not constrained in a linear fit to the (semi)leptonic kaon decay data, and whose quadratic terms represent the leading NP contributions to the $K_{e 3}$ differential distributions. Indeed, this has been used by the ISTRA [64] and NA48 [65] Collaborations to set bounds on those WC. However, none of these fits contain simultaneously the four relevant quantities, namely, the leading SM form-factors parameters $\lambda_{+}^{\prime}, \lambda_{+}^{\prime \prime}$ and both WC $\epsilon_{S, T}^{s e}$, and the correlations are not given either. The strongest bounds were obtained by ISTRA [64] in fits to $\lambda_{+}, \lambda_{+}^{\prime}$ and one of the $\mathrm{WC}$ at a time, finding

$$
\begin{aligned}
& \frac{f_{S}}{f_{+}(0)}=\frac{m_{K}^{2}-m_{\pi}^{2}}{2 m_{K}\left(m_{s}-m_{u}\right)} \epsilon_{S}^{s e}=-0.004\left({ }_{-6}^{+7}\right)(4), \\
& \frac{f_{T}}{f_{+}(0)}=-2 \frac{B_{T}(0)}{f_{+}(0)} \epsilon_{T}^{s e}=-0.012(21)(11),
\end{aligned}
$$

where we have transformed the results in terms of the variables employed in that work, $f_{T} / f_{+}(0)$ and $f_{T} / f_{+}(0)$, into those employed in this work.

On the other hand, as discussed in section 3.1, $\epsilon_{P}^{D \ell}$ contributes to $P_{\ell 2}$ with a large helicity-enhancement that can make their quadratic terms important too. In fact, this quadratic contribution allows for a second solution, different from zero, to the constraint that can be obtained on the pseudoscalar WC. To be more explicit, this occurs when 


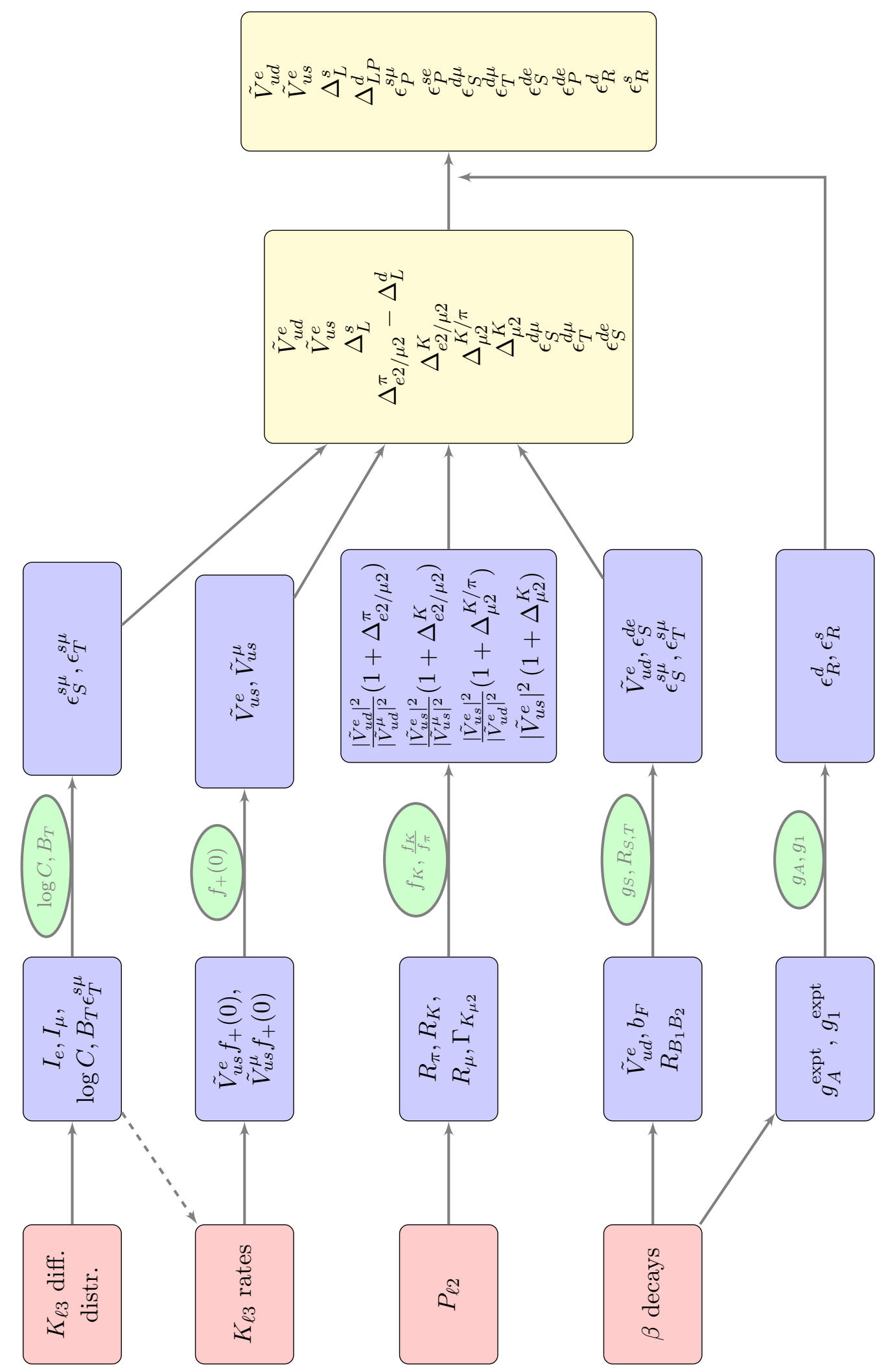

Figure 1. Flowchart. 


\begin{tabular}{|c|c|c|}
\hline$P_{\ell 2(\gamma)}$ & $K_{\ell 3(\gamma)}$ & Baryon $\beta$-decay \\
\hline$R_{\pi}=1.2344(30) \times 10^{-4}[94]$ & {$\left[\tilde{V}_{u s}^{e} f_{+}(0)\right]_{\epsilon_{T}=0}=0.21649(44)$} & $\left|\tilde{V}_{u d}^{e}\right|=0.97451(38)$ \\
$R_{K}=2.488(9) \times 10^{-5}[4]$ & {$\left[\tilde{V}_{u s}^{\mu} f_{+}(0)\right]_{\epsilon_{T}=0}=0.21667(54)$} & $b_{f}=-0.0028(26)[74]$ \\
$\Gamma\left(K_{\mu 2}\right)=5.134(10) \times 10^{7} s^{-1}$ & $\log C=0.1985(70)[43]$ & $g_{A}=-1.2723(23)[94]$ \\
$\operatorname{BR}\left(\pi_{\mu 2}\right)=0.9998770(4)[4]$ & $-2 \epsilon_{T}^{s \mu} \frac{B_{T}(0)}{f_{+}(0)}=-0.0007(71)[63]$ & $\left.\frac{g_{1}}{f_{1}}\right|_{\Lambda p}=0.718(15)[4]$ \\
$\tau_{\pi^{ \pm}}=2.6033(5) \times 10^{-8} s[4]$ & & \\
\hline
\end{tabular}

Table 1. Experimental data used in the analysis. See main text for more details about those values that do not have a reference in the table. Additionally, the various masses and $\alpha_{\mathrm{em}}$ are taken from the PDG [4].

$1-\left(B_{0} / m_{\ell}\right) \epsilon_{P}^{D \ell} \simeq-1$, namely for $\epsilon_{P}^{D e} \sim 4 \times 10^{-4}$ or $\epsilon_{P}^{D \mu} \sim 0.1$. In order to discard this other solution one would need an independent constraint on these WC that could be provided by $\beta$ decays $[76,89]$. Finally note that if we allow for a complex CP-violating phase in the WC these two-fold degeneracies become circular in the complex plane of $\epsilon_{P}^{D \ell}[47,51] .6$

Finally, for completeness, we discuss the subleading operators $(D>6)$ in the lowenergy EFT, i.e. operators with derivatives neglected in the effective Lagrangian of eq. (2.1). These terms are corrections of order $(q / v)^{n} \lesssim\left(M_{K} / v\right)^{n} \sim\left(10^{-3}\right)^{n}(n \geq 1)$ with respect to the non-derivative terms. In the SM they are generated with $n=2$ at tree level (NLO terms in the $\mathrm{W}$-propagator expansion) and are thus still unobservable in beta decays. An example of NP giving this type of contributions (with $n=1)$ are the dipole-type $(D=6)$ $\mathrm{SU}(2) \times \mathrm{U}(1)$-invariant operators such as $\left(\bar{\ell} \sigma^{\mu \nu} e\right) \tau^{I} \varphi W_{\mu \nu}^{I}$. Their effect will be then of order $10^{-3} \times v^{2} / \Lambda^{2}$, which will be unobservable even for NP scales not much larger than the EW scale.

\section{Phenomenology}

\section{$5.1 \quad$ Inputs}

\subsubsection{Experimental}

We summarize in table 1 the experimental values used for our analysis. Now we discuss some non-trivial aspects of them:

- We calculate $\Gamma\left(K_{\mu 2}\right)$ using the latest Flavianet results for the corresponding BR and the lifetime [43], and taking into account their 10\% correlation [95].

- $\boldsymbol{K}_{\ell \mathbf{3}}$ shapes: We take the value of $\log C$ from the latest Flavianet update [43] and the tensor term for the muonic mode from the ISTRA+ analysis [63]. We assume these bounds will hold in a combined fit, and we neglect the correlations of $\log C$,

\footnotetext{
${ }^{6}$ Any chiral-enhanced not-interfering term has the same consequences, e.g. operators with light righthanded [51, 92, 93] or "wrong-flavor" neutrinos [47].
} 
the tensor term and the phase-space integrals $I_{K, 0}^{\ell}$. It should be straightforward to amend these limitations by the experimental collaborations.

- $\boldsymbol{K}_{\ell \mathbf{3}}$ rates: We take the latest Flavianet results [43] for the product $\left|\tilde{V}_{u s}^{\ell}\right| f_{+}(0)$ obtained from the channels $K_{L e 3}, K_{L \mu 3}, K_{S e 3}, K_{e 3}^{ \pm}, K_{\mu 3}^{ \pm}$, and taking into account their correlations [95] we average them to obtain the values $\left|\tilde{V}_{u s}^{e}\right| f_{+}(0)$ and $\left|\tilde{V}_{u s}^{\mu}\right| f_{+}(0)$ shown in table 1 , which present a $+52 \%$ correlation. Since the Flavianet extraction sets to zero the tensor term, these numbers are still missing the contribution from the tensor phase space integral, cf. eqs. (3.20)-(3.21), which we denote in table 1 with the subindex " $\epsilon_{T}=0$ ". Combining these values with the bound on the tensor term, we find

$$
\left(\begin{array}{c}
\left|\tilde{V}_{u s}^{e}\right| f_{+}(0) \\
\left|\tilde{V}_{u s}^{\mu}\right| f_{+}(0) \\
\epsilon_{T}^{s \mu} \frac{B_{T}(0)}{f_{+}(0)}
\end{array}\right)=\left(\begin{array}{c}
0.21649(44) \\
0.21670(59) \\
-0.0007(71)
\end{array}\right), \quad \rho=\left(\begin{array}{ccc}
1.0 .47 & 0 . \\
- & 1 . & -0.42 \\
- & - & 1 .
\end{array}\right),
$$

where we have simply used that the total phase space integral is given by

$$
I_{K}^{\mu} \approx I_{K, 0}^{\mu}\left(1-0.40 \epsilon_{T}^{s \mu}\right)
$$

using the Flavianet determination of $I_{K, 0}^{\mu}$ and $B_{T}(0) / f_{+}(0)$ from ref. [96].

- Nuclear $\boldsymbol{\beta}$ decays: current studies of superallowed nuclear transitions contain a SM analysis where $V_{u d}$ is extracted, finding $\left|\tilde{V}_{u d}^{e}\right|=0.97417(21)$, and a NP analysis where the Fierz term $b_{F}$ is bounded, $b_{F}=-0.0028(26)$ [74]. In our framework we need a combined extraction of both quantities. Assuming a Gaussian $\chi^{2}$ we can reconstruct the outcome of such a fit. For this reconstruction we use that the minimum of the 2-parameter fit is $\left\{\mathcal{F} t, b_{F}\right\}=\{3070.1 s,-0.0028\}$ [97], where $\mathcal{F} t$ is the so-called "corrected" $\mathcal{F} t$ value, where different nuclear-structure and transitiondependent radiative corrections have been subtracted (see e.g. ref. [74]). We obtain:

$$
\left(\begin{array}{c}
\left|\tilde{V}_{u d}^{e}\right| \\
b_{F}
\end{array}\right)=\left(\begin{array}{c}
0.97451(38) \\
-0.0028(26)
\end{array}\right), \rho=\left(\begin{array}{cc}
1 . & -0.83 \\
- & 1 .
\end{array}\right) .
$$

These results should be taken with caution, keeping in mind their reconstructed nature. Ideally, in the future they will be given in this format, where it is trivial to recover the SM limit setting $b_{F}=0$. Let us remind that $b_{F}=-2 g_{S} \epsilon_{S}^{d e}$, where $g_{S}$ is the corresponding scalar form factor (see e.g. ref. [89]).

- Neutron $\boldsymbol{\beta}$ decays: We use the PDG average for the axial charge $g_{A}$. Let us notice that this determination, which comes typically from the measurement of the neutron $\beta$ asymmetry $A$, assumes the SM is correct, whereas in our EFT framework it provides a $g_{A}$ value modified by scalar and tensor interactions (in addition to $\epsilon_{R}^{d}$ ). However, given the current bounds on $\epsilon_{S, T}^{d e}$, these effects can be neglected in comparison with the error of the lattice $g_{A}$ determination, which by far limits the $\epsilon_{R}^{d}$ bound. And the same applies to the hyperon axial charge $g_{1}$. 


\begin{tabular}{|c|c|c|}
\hline$P_{\ell 2(\gamma)}$ & $K_{\ell 3(\gamma)}$ & Baryon $\beta$-decay \\
\hline$R_{\pi}^{\mathrm{SM}}=1.2352(1) \times 10^{-4}[17]$ & $f_{+}(0)=0.9661(32)[5]$ & $g_{S}=1.02(11)[89]$ \\
$R_{K}^{\mathrm{SM}}=2.477(1) \times 10^{-5}[17]$ & $\Delta_{\mathrm{CT}}=-0.0035(80)[2,8]$ & $g_{A}=1.24(4)[85]$ \\
$f_{K^{ \pm}} / f_{\pi^{ \pm}}=1.192(5)[5]$ & $\frac{B_{T}(0)}{f_{+}(0)}=0.68(3)[96]$ & $\left.\frac{g_{1}}{f_{1}}\right|_{\Lambda p}=0.72(7)$ \\
$f_{K^{ \pm}}=154.3(0.4)(2.8) \mathrm{MeV}[98]$ & & \\
$\delta_{\mathrm{em}}^{K \mu}-\delta_{\mathrm{em}}^{\pi \mu}=-0.0069(17)$ & & \\
$\delta_{\mathrm{em}}^{K \mu}=0.0121(32)$ & & \\
\hline
\end{tabular}

Table 2. Theory inputs. See main text for more details about those values that do not have a reference in the table. The scale/scheme-dependent quantities are given in the $\overline{M S}$ at $\mu=2 \mathrm{GeV}$.

- Hyperon $\beta$ decays: in contrast to $g_{A}$, the axial-charges in the hyperons decays, $g_{1}$, are measured with a relative uncertainty not better than $2 \%$ [4]. The best precision is achieved in $\Lambda \rightarrow p e^{-} \bar{\nu}$ that we will use as a reference for the extraction of $\epsilon_{R}^{s}$ in the fits. As discussed in section 3.4, the hyperon decays also provide independent limits on the scalar and tensor muonic WC from the lepton-universality ratio in eq. (3.26). The current bound on the tensor WC, $\epsilon_{T}^{s \mu}=-0.017(20)(1 \sigma)[76],{ }^{7}$ is only 4 times less precise than the one obtained from the shapes of $K_{\mu 3}$ and does not depend on the assumptions described above for this mode.

\subsubsection{Theoretical}

We summarize in table 2 the theory input for our analysis. Some comments are in order:

- Using the expression in eq. (3.2), we find $\delta_{\mathrm{em}}^{K \mu}=0.0121(12)_{c_{1}^{K}}(30)_{e^{2} p^{4}}$ for the EM corrections to $K_{\mu 2(\gamma)}$ at order $e^{2} p^{2}$ in the chiral expansion. The first error comes from the uncertainty in the hadronic structure constant $c_{1}^{K}=-1.98(50)[15,17]$ at order $e^{2} p^{2}$ and the second error (25\% of the central value) is an estimate of corrections due to higher order terms. Likewise we find $\delta_{\mathrm{em}}^{K \mu}-\delta_{\mathrm{em}}^{\pi \mu}=-0.0069(17)_{e^{2} p^{4}}$, which is free of $c_{1}^{P}$ uncertainties at this order.

- We use FLAG averages $\left(N_{f}=2+1\right)[5]$ for $f_{K^{ \pm}} / f_{\pi^{ \pm}}[99-101]$ and $f_{+}(0)[102,103]$.

- The experimental value of $f_{\pi}$ is often used to set the scale in the LQCD calculations. Within the SMEFT setup this is not convenient because one propagates the NP contribution $\Delta_{\mu 2}^{\pi}$ onto all the dimensionful quantities determined thereafter in the lattice. Namely, the corresponding determination of $f_{K}$ makes $\Gamma\left(K_{\mu 2(\gamma)}\right)$ not sensitive to $\Delta_{\mu 2}^{K}$, but only to $\Delta_{\mu 2}^{K / \pi}$, i.e. the direction already probed by the $R_{\mu}$ ratio. Thus, it is better to use determinations where an observable dominated by strong dynamics is used to set the scale. Among the determinations passing the FLAG requirements [5, 98, 99, 101] we choose for our fit the MILC09 calculation as it already includes the

\footnotetext{
${ }^{7}$ Here we take into account that the $\epsilon_{T}^{s \mu}$ definition of ref. [76] has a minus sign difference with the definition used in this work.
} 
isospin corrections, $f_{K^{ \pm}}=154.3(0.4)(2.8) \mathrm{MeV}$ [98]. Notice that this caveat also holds in a global SM analysis of $K_{\ell 2}$ and $\pi_{\ell 2}$ data, since using the experimental value of $f_{\pi}$ to set the QCD scale entails the loss of one of the experimental inputs.

- Using the values in the table 2 we find $\log C_{\mathrm{QCD}}=0.2073(84)$. The correlation between this number and the quantities $f_{K^{ \pm}} / f_{\pi^{ \pm}}$and $f_{+}(0)$ is taken into account.

- There are a few recent $N_{f}=2 \mathrm{LQCD}$ calculations of axial charge of the nucleon at the physical point [85-87]. We use $g_{A}=1.24(4)$ [85].

- There are no computations of the axial charges for the semileptonic $\Delta S=1$ hyperon decays in the lattice yet, although pioneering calculations of the $\Delta S=0$ ones of the $\Sigma$ and $\Xi$ baryons have been reported [104-106]. Taking the results in ref. [105] (and $g_{A}$ from ref. [85]), we obtain $g_{\Sigma}=0.91(4)$ and $g_{\Xi}=-0.25(3)$. These can be connected to the $\Delta S=1$ couplings using $\mathrm{SU}(3)_{F}$, which is known to work empirically at few-percent accuracy for these quantities (see discussions in refs. [88, 107]). We obtain $g_{1} /\left.f_{1}\right|_{\Lambda p}=0.72(7)$, where we have conservatively estimated SU(3)-breaking corrections by a $10 \%$. Needless to say that the situation could be improved with direct LQCD calculations of these couplings.

\section{$5.2 \quad$ Fit}

Using the experimental and theoretical inputs listed in tables 1 and 2, and treating all errors as Gaussian, we perform a standard $\chi^{2}$ fit, keeping only linear terms in $v^{2} / \Lambda^{2}$ in the theoretical expressions. The results are:

$$
\left(\begin{array}{c}
\tilde{V}_{u d}^{e} \\
\tilde{V}_{u s}^{e} \\
\Delta_{L}^{s} \\
\Delta_{L P}^{d} \\
\epsilon_{P}^{d e} \\
\epsilon_{R}^{d} \\
\epsilon_{P}^{s e} \\
\epsilon_{P}^{s \mu} \\
\epsilon_{R}^{s} \\
\epsilon_{S}^{s \mu} \\
\epsilon_{T}^{s \mu} \\
\epsilon_{S}^{d e}
\end{array}\right)=\left(\begin{array}{c}
0.97451 \pm 0.00038 \\
0.22408 \pm 0.00087 \\
1.0 \pm 2.5 \\
1.9 \pm 3.8 \\
4.0 \pm 7.8 \\
-1.3 \pm 1.7 \\
-0.4 \pm 2.1 \\
-0.7 \pm 4.3 \\
0.1 \pm 5.0 \\
-3.9 \pm 4.9 \\
0.5 \pm 5.2 \\
1.4 \pm 1.3
\end{array}\right) \times 10^{\wedge}\left(\begin{array}{c}
0 \\
0 \\
-3 \\
-2 \\
-6 \\
-5 \\
-3 \\
-2 \\
-4 \\
-3 \\
-3
\end{array}\right),
$$

in the $\overline{M S}$ scheme at $\mu=2 \mathrm{GeV}$. Let us remind the reader that $\Delta_{L}^{s}=\epsilon_{L}^{s \mu}-\epsilon_{L}^{s e}$ and $\Delta_{L P}^{d}=\epsilon_{L}^{d e}-\epsilon_{L}^{d \mu}+\frac{B_{0}}{m_{\mu}} \epsilon_{P}^{d \mu} \approx \epsilon_{L}^{d e}-\epsilon_{L}^{d \mu}+24 \epsilon_{P}^{d \mu}$. 
The correlation matrix is given by:

$$
\rho=\left(\begin{array}{ccccccccccc}
1.0 . & 0 . & 0.01 & 0.01 & 0 . & 0 . & 0 . & 0 . & 0 . & 0 . & 0.82 \\
-1 . & -0.16 & 0 . & 0 . & 0 . & 0.04 & 0.04 & 0 . & -0.26 & 0 . & 0 . \\
-- & 1 . & 0 . & 0 . & 0 . & -0.01 & 0.02 & 0 . & 0 . & 0.46 & 0 . \\
-- & - & 1 . & 0.9995 & -0.87 & 0.09 & 0.09 & 0 . & 0.04 & 0 . & 0.01 \\
-- & - & - & 1 . & -0.87 & 0.09 & 0.09 & 0 . & 0.04 & 0 . & 0.01 \\
-- & - & - & - & 1 . & 0 . & 0 . & 0 . & 0 . & 0 . & 0 . \\
-- & - & - & - & - & 1 . & 0.9995 & -0.98 & -0.01 & 0 . & 0 . \\
-- & - & - & - & - & - & 1 . & -0.98 & -0.01 & 0.01 & 0 . \\
-- & - & - & - & - & - & - & 1 . & 0 . & 0 . & 0 . \\
-- & - & - & - & - & - & - & - & 1 . & 0 . & 0 . \\
-- & - & - & - & - & - & - & - & - & 1 . & 0 . \\
-- & - & - & - & - & - & - & - & - & - & 1 .
\end{array}\right) .
$$

Exploiting now the unitarity of the CKM matrix as explained in section 4, trading $\left|\tilde{V}_{u s}^{e}\right|$ for $\Delta_{\mathrm{CKM}}$, we obtain:

$$
\begin{aligned}
& \Delta_{\mathrm{CKM}}=2\left|V_{u d}^{e}\right|^{2}\left(\epsilon_{L}^{d e}+\epsilon_{R}^{d}\right)+2\left|V_{u s}\right|^{2}\left(\epsilon_{L}^{s e}+\epsilon_{R}^{s}\right)-2 \frac{\delta G_{F}}{G_{F}} \\
& =-(1.2 \pm 8.4) \times 10^{-4} \text {, }
\end{aligned}
$$

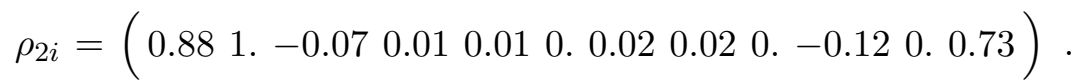

We observe good agreement with the SM, with marginalized limits varying from the $10^{-5}$ level for the pseudoscalar couplings in the electronic channel (due to the chiral enhancement) to the per-cent level for the right-handed couplings (due to the limited lattice precision in the axial-vector form factors).

We observe also that the combinations of $\mathrm{WC}\left\{\Delta_{L P}^{d}, \epsilon_{P}^{d e}\right\}$ and $\left\{\epsilon_{P}^{s e}, \epsilon_{P}^{s \mu}\right\}$ are highly correlated, which simply reflects the fact that the specific combination of them that appears in $R_{\pi}$ and $R_{K}$ respectively is much more constrained than the individual WC. This is illustrated by the limits obtained when the only non-zero NP couplings are the pseudoscalar couplings in the electronic channel:

$$
\begin{aligned}
& \epsilon_{P}^{d e}=(0.7 \pm 2.5) \times 10^{-7}, \\
& \epsilon_{P}^{s e}=-(4.5 \pm 3.7) \times 10^{-7} .
\end{aligned}
$$

Such strong bounds can be the result of a very high NP scale, $\Lambda \sim v / \sqrt{\epsilon} \sim \mathcal{O}(500) \mathrm{TeV}$, or a non-trivial structure in lepton-flavor space, such as $\epsilon_{P}^{D \ell} \sim m_{\ell} \epsilon_{P}^{D}$. The latter case naturally follows in models with extra Higgs doublets [1] or, model-independently, from scalar fourfermion operators with Minimal Flavor Violation (MFV) [108-112] as in ref. [46].

We complete the numerical discussion with the uncorrelated bounds that are obtained from $\pi_{e 2 \gamma}$, eq. (3.10), and including quadratic effects in the Dalitz plots of $K_{e 3}$, eq. (4.7):

$$
\begin{aligned}
\epsilon_{T}^{d e} & =(0.1 \pm 0.8) \times 10^{-3}, \\
\epsilon_{S}^{s e} & =(-1.6 \pm 3.3) \times 10^{-3}, \\
\epsilon_{T}^{s e} & =(0.9 \pm 1.8) \times 10^{-2},
\end{aligned}
$$

in the $\overline{M S}$ scheme at $\mu=2 \mathrm{GeV}$. Here we have used the result in table 2 for $B_{T}(0) / f_{+}(0)$. 


\subsubsection{The $V_{u d}-V_{u s}$ plane revamped}

An application that particularly highlights the virtues of the EFT framework developed in this work is the $\left|V_{u s}\right|-\left|V_{u d}\right|$ plot that illustrates the consistency of $K_{\ell 3}, K_{\ell 2} / \pi_{\ell 2}$ and nuclear- $\beta$-decay determinations [91]. Interestingly enough, although the values of $\left|V_{u d}\right|$ and $\left|V_{u s}\right|$ currently extracted from nuclear $\beta$ decays an $K_{\ell 3}$ are in perfect agreement with unitarity, a small "misalignment" with the $K_{\ell 2} / \pi_{\ell 2}$ bound is observed [5, 6, 43].

In the general EFT setup, and if we focus on the electronic channel, this plot represents a projection of the global fit discussed earlier into the $\left|\tilde{V}_{u d}^{e}\right|-\left|\tilde{V}_{u s}^{e}\right|$ plane. The NP can manifest either as a violation of CKM unitarity or, precisely, as this misalignment of the bound on $\left|\tilde{V}_{u s}^{e}\right| /\left|\tilde{V}_{u d}^{e}\right|$ from $K_{e 2} / \pi_{e 2}$ with respect to the intersection of the other two bounds from $\beta$ decays $\left(\left|\tilde{V}_{u d}^{e}\right|\right)$ and $K_{e 3}\left(\left|\tilde{V}_{u s}^{e}\right|\right)$. The former case corresponds to the bound on $\Delta_{\mathrm{CKM}}$ obtained above, whereas the latter probes the combination of Wilson Coefficients $\Delta_{e}^{K / \pi} / 2=$ $-2\left(\epsilon_{R}^{s}-\epsilon_{R}^{d}\right)-\frac{B_{0}}{m_{e}}\left(\epsilon_{P}^{s e}-\epsilon_{P}^{d e}\right)$, cf. eqs. (3.7)-(3.8). Hence, the right-handed and pseudoscalar contributions change the slope of the diagonal constraint obtained from $K_{e 2} / \pi_{e 2}$.

In figure 2 we show current experimental constraints on the $\left|\tilde{V}_{u d}^{e}\right|-\left|\tilde{V}_{u s}^{e}\right|$ plane, where we have added a NP contribution $\Delta_{e 2}^{K / \pi} \simeq 0.02$ needed to perfectly align the band from $K_{e 2} / \pi_{e 2}$ with those from $\beta$-decays and $K_{e 3}$. For illustration, we also show the diagonal lines corresponding to a NP contribution in $\Delta_{e 2}^{K / \pi}$ if it was of the type $\epsilon_{P}^{s e}$ for different values of the corresponding effective scale $\Lambda_{P}^{s e}$.

Nonetheless, this effect has a small significance, as reflected by the consistency of the data with the SM in our global fit discussed in the previous section. We see that this precise test of the SM, obtained thanks to the small experimental and theoretical uncertainties achieved in these processes, currently allows one to probe $\mathcal{O}(100) \mathrm{TeV}$ scales.

Let us stress that figure 2 is obtained from our global fit, with all NP terms present, which makes the horizontal and vertical error bands wider. The traditional $V_{u d}-V_{u s}$ plot is recovered if the only NP terms present are those probed in this plot, i.e. $\Delta_{\mathrm{CKM}}$ and $\Delta_{\mu 2}^{K / \pi}$. That allows one to combine $K_{e 3}$ and $K_{\mu 3}$ SM extractions of $\tilde{V}_{u s}$ and to use the SM analysis of ref. [74] for $V_{u d}$, cf. eq. (5.3). In that limit, the sensitivity to $\Delta_{\mathrm{CKM}}$ and $\Delta_{\mu 2}^{K / \pi}$ is of course stronger, and a larger (though still not significant) tension arises in the plot $[5,6,43]$.

Needless to say, one could have plotted instead the bound obtained from $K_{\mu 2} / \pi_{\mu 2}$, with the only difference that the combination of WC probed in that case is longer, involving also left-handed $\epsilon_{i}$ to connect $\tilde{V}_{u D}^{\mu}$ with $\tilde{V}_{u D}^{e}$. And the same applies e.g. for the $K_{\mu 2}$ extraction.

\subsection{Minimal Flavor Violation and SM limits}

If the flavor symmetry $\mathrm{U}(3)^{5}$ is respected, all NP terms vanish except those contaminating the CKM matrix elements, which in this case become lepton-independent [38]. This NP flavor structure occurs if flavor breaking is suppressed by a mechanism such as Minimal Flavor Violation. Thus, the MFV analysis and the SM one (without imposing CKM unitarity) are equivalent. In this case we find:

$$
\left(\begin{array}{c}
\tilde{V}_{u d} \\
\tilde{V}_{u s}
\end{array}\right)=\left(\begin{array}{c}
0.97416(21) \\
0.22484(64)
\end{array}\right), \quad \rho=\left(\begin{array}{c}
1.0 .03 \\
-1 .
\end{array}\right),
$$




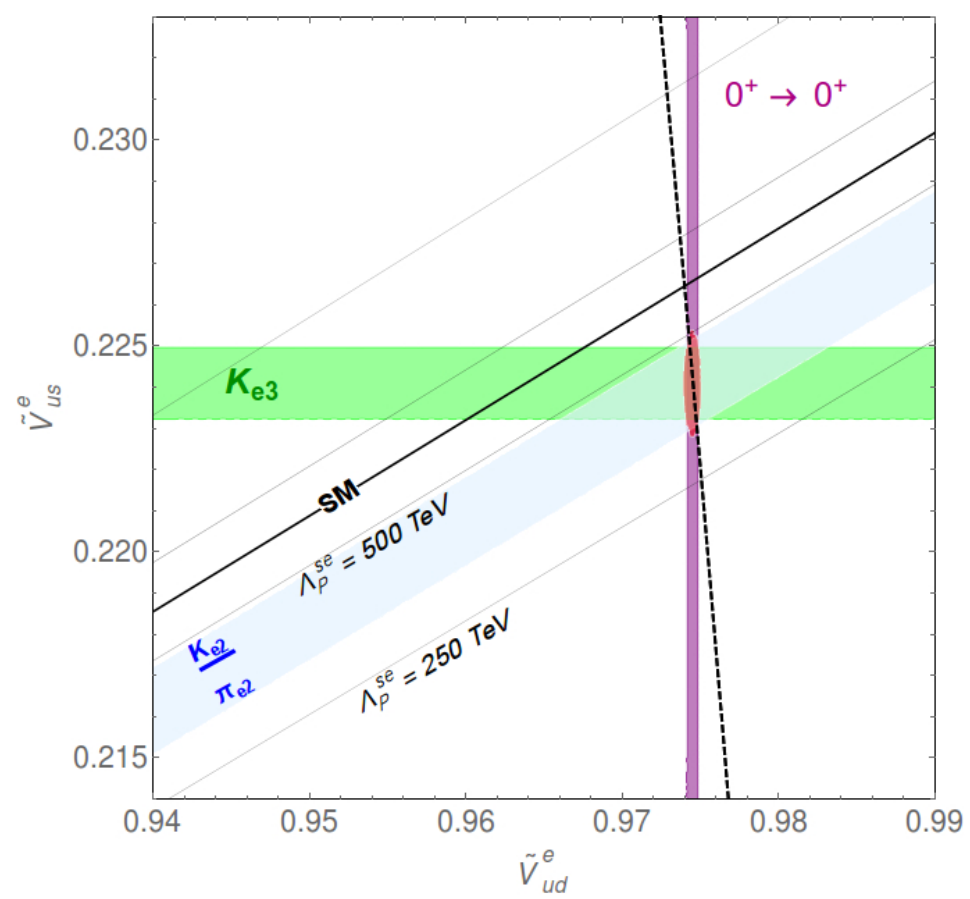

Figure 2. $1 \sigma$ regions for $\left|\tilde{V}_{u d}^{e}\right|$ and $\left|\tilde{V}_{u s}^{e}\right|$ from $K_{e 3}$ (horizontal band) and nuclear $\beta$ decays (vertical band). We also plot the $1 \sigma$ region given by the ratio $\Gamma\left(K_{e 2(\gamma)}^{ \pm}\right) / \Gamma\left(\pi_{e 2(\gamma)}^{ \pm}\right)$(diagonal band) assuming a NP contribution $\Delta_{e 2}^{K / \pi} \simeq 0.02$, along with the lines corresponding to different NP effective scales $\Lambda_{P}^{s e} \approx\left(V_{u s} \epsilon_{P}^{s e}\right)^{-1 / 2} v$. The dashed black line shows the CKM unitarity constraint.

where the tildes in the left-hand side apply only to the MFV case. The only NP probe left is then the CKM unitarity test [38]:

$$
\Delta_{\mathrm{CKM}}=-(4.6 \pm 5.2) \times 10^{-4} .
$$

In a SM analysis where the CKM unitarity is imposed, this NP term is set to zero, reducing the error in the matrix elements:

$$
\left|V_{u d}\right|=0.97432(12) \quad \text { or equivalently } \quad\left|V_{u s}\right|=0.2252(5) .
$$

Last but not least, we stress that our fit contains also the various QCD quantities as outputs. In the general EFT case, they are trivially equal to their lattice QCD values that we use as inputs, since the fit is not overdetermined. On the other hand, this is not the case in the SM limit, where experimental data do complement the lattice calculations [2], giving

$$
\left(\begin{array}{c}
f_{K^{ \pm}} \\
f_{K^{ \pm}} / f_{\pi^{ \pm}} \\
f_{+}(0)
\end{array}\right)=\left(\begin{array}{c}
155.62(44) \mathrm{MeV} \\
1.1936(30) \\
0.9632(23)
\end{array}\right), \quad \rho=\left(\begin{array}{ccc}
1 . & 0.78 & 0.56 \\
- & 1 . & 0.64 \\
- & - & 1 .
\end{array}\right) .
$$

Figure 3 shows these results, and compare them with the bounds obtained using only LQCD [5] or only experimental data, finding a good agreement.

The framework developed in this work is explicitly designed to make use of all the experimental information available in (semi)leptonic kaon and pion decays. In addition to 


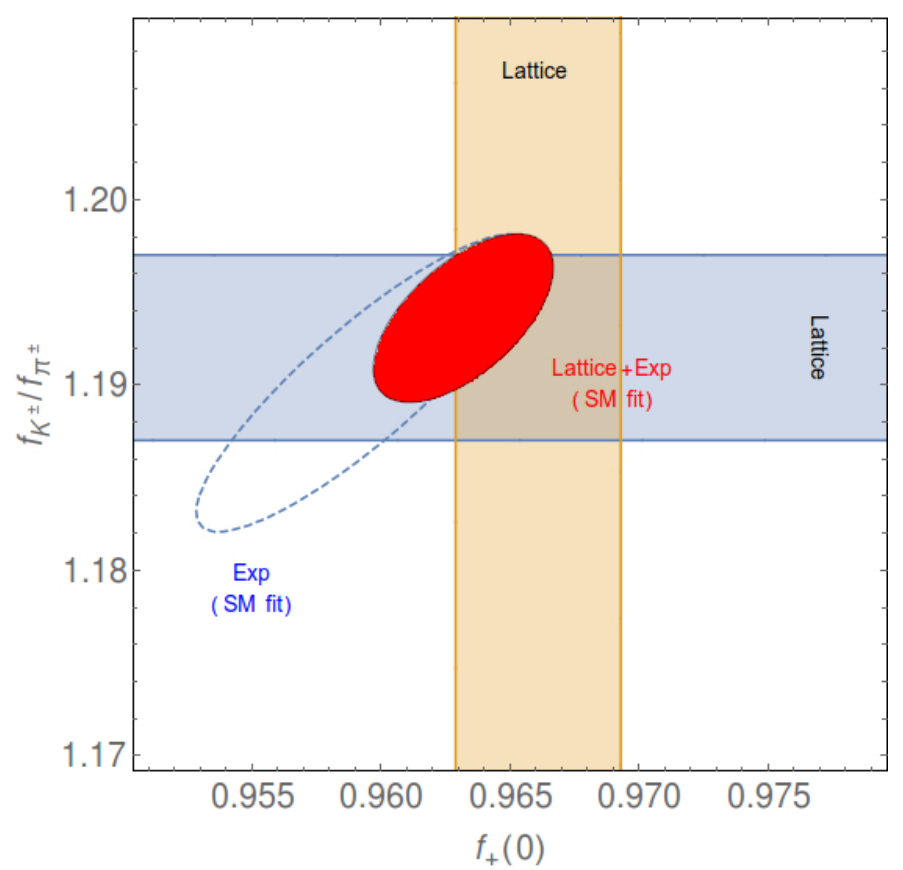

Figure 3. $68 \%$ C.L. regions for $f_{K^{ \pm}} / f_{\pi^{ \pm}}$and $f_{+}(0)$ using only LQCD [5], only experimental data (in a SM fit) or using both.

\begin{tabular}{|ccccc|}
\hline Analysis & $V_{u s}$ & Data & Form Factors & $K_{\mu 2(\gamma)}$ and CTT \\
\hline This work & $0.22484(64)$ & $2014[43]$ & $2013[5]$ & yes \\
\hline $\begin{array}{c}\text { Moulson'2014 [43] } \\
\text { (our code) }\end{array}$ & $0.2248(7)$ & $2014[43]$ & $2013[5]$ & no \\
\hline FLAG'2013 [5] & $0.2248(7)$ & & & no \\
(our code) & $0.2247(7)$ & $2010[2]$ & $2013[5]$ & no \\
\hline $\begin{array}{c}\text { Flavianet'2010 [2] } \\
\text { (our code) }\end{array}$ & $0.2253(9)$ & $2010[2]$ & $2010[2]$ & \\
\hline
\end{tabular}

Table 3. Comparison with other SM analyses (without imposing CKM unitarity and using $N_{f}=$ $2+1$ lattice calculations).

the usual input of any analysis where $V_{u d}$ and $V_{u s}$ are extracted (that is, $K_{\ell 3}, K_{\mu 2} / \pi_{\mu 2}$ and $\beta$ decays), our fit takes into account two more pieces of information. First, it includes the $K_{\mu 2(\gamma)}$ rate that, along with a lattice determination for $f_{K^{ \pm}}$, offers an additional handle on $V_{u s}$. Secondly, we incorporate the CTT constraint in our fit, which, combined with the experimental determination of $\log C$, provides an extra handle on $f_{K^{ \pm}} / f_{\pi^{ \pm}}$and $f_{+}(0)$; namely $f_{K^{ \pm}} / f_{\pi^{ \pm}} \times 1 / f_{+}(0)=1.223(12)$. Indeed, this is part of the information that made possible the determinations shown in eq. (5.13). Although the CTT has been used before as an LQCD/ChPT check and as a NP probe (see e.g. ref. [2]), it was not taken into account in the $V_{u D}$ extractions. 
As a consistency check, we have used our fitting code with the inputs of a few wellknown SM analyses and, as shown in table 3, we reproduce very well their corresponding results. The only difference between our SM analysis and that of ref. [43] is the inclusion of the additional inputs discussed above, i.e. $K_{\mu 2(\gamma)}$ and the CTT. Thus, the comparison with it shows clearly that their numerical impact on the $V_{u d}$ and $V_{u s}$ values is very small. This is due to the not-precisely-enough values of $f_{K^{ \pm}}, \log C_{\text {expt }}$ and $\Delta_{\mathrm{CT}}$. In fact, with the lattice values used in this work we find $f_{K^{ \pm}} / f_{\pi^{ \pm}} \times 1 / f_{+}(0)=1.234(7)$. Let us stress that the use of $K_{\mu 2(\gamma)}$ and CTT was, however, critical in the general NP analysis presented in the previous section.

\section{SMEFT and complementarity with collider searches}

In order to connect the experimental bounds on the WC obtained at low-energies with those generated by NP models at a high-energy scale, one needs to take into account the running and mixing under radiative corrections of the corresponding operators.

At the same time, such scale evolution allows one to make contact with the high-energy SMEFT. Employing this EFT as in intermediate step before connecting to specific models is convenient for several reasons. First, it is constrained by the more restrictive EW gauge symmetry group which leads to model-independent relations among the WC that are not present in the low-energy analysis (viz. eq. (2.3)) [38, 44]. In addition, and due again to $\mathrm{SU}(2) \times \mathrm{U}(1)$, the SMEFT Wilson Coefficients enter not only in the processes studied in this work but also in other low-energy charged-current or neutral current processes involving first- and second- generation fermions, so that an interesting degree of complementarity is expected with charm-hadron (semi)leptonic decays, rare kaon decays, etc.

Last but not least, the SMEFT makes possible to study model-independently the interplay between the low-energy measurements discussed in this work and NP searches at colliders. Although such studies are clearly beyond the scope of this work, in this section we show the potential of this approach through some illustrative and simple examples.

\subsection{RGE running and matching to the SMEFT}

As explained in section 2, (pseudo)scalar and tensor WC run under QCD and, moreover, they mix through EW interactions. We take both effects into account integrating the coupled differential renormalization group equations:

$$
\frac{d \vec{\epsilon}(\mu)}{d \log \mu}=\left(\frac{\alpha(\mu)}{2 \pi} \gamma_{\mathrm{ew}}+\frac{\alpha_{s}(\mu)}{2 \pi} \gamma_{s}\right) \vec{\epsilon}(\mu)
$$

where, once again, $\vec{\epsilon}(\mu)=\left(\epsilon_{S}^{D \ell}(\mu), \epsilon_{P}^{D \ell}(\mu), \epsilon_{T}^{D \ell}(\mu)\right)$. Evolving from the low-energy scale $\mu=2 \mathrm{GeV}$ to a typical LHC scale such as $\mu=1 \mathrm{TeV}$, we find

$$
\left(\begin{array}{c}
\epsilon_{S}^{D \ell} \\
\epsilon_{P}^{D \ell} \\
\epsilon_{T}^{D \ell}
\end{array}\right)_{(\mu=1 \mathrm{TeV})}=\left(\begin{array}{ccc}
0.51 & -0.0014 & 0.35 \\
-0.0014 & 0.51 & 0.35 \\
0.0031 & 0.0031 & 1.08
\end{array}\right)\left(\begin{array}{c}
\epsilon_{S}^{D \ell} \\
\epsilon_{P}^{D \ell} \\
\epsilon_{T}^{D \ell}
\end{array}\right)_{(\mu=2 \mathrm{GeV})}
$$


where one can see an important mixing between tensor and (pseudo)scalar, which is simply the result of having large coefficients in the corresponding entries of the electroweak anomalous dimension matrix of eq. (2.8).

These results can be trivially used to run the bounds to the high scale:

$$
\left(\begin{array}{c}
\epsilon_{S}^{d e} \\
\epsilon_{P}^{d e} \\
\epsilon_{T}^{d e} \\
\epsilon_{S}^{s e} \\
\epsilon_{P}^{s e} \\
\epsilon_{T}^{s e} \\
\epsilon_{S}^{s \mu} \\
\epsilon_{P}^{s \mu} \\
\epsilon_{T}^{d \mu}
\end{array}\right)=\left(\begin{array}{c}
7.4 \pm 7.1 \\
0.3 \pm 2.8 \\
1.1 \pm 8.7 \\
2.3 \pm 6.5 \\
3.1 \pm 6.2 \\
1.0 \pm 1.9 \\
0.0 \pm 1.8 \\
-0.2 \pm 2.9 \\
0.6 \pm 5.6
\end{array}\right) \times 10^{\wedge}\left(\begin{array}{c}
-4 \\
-4 \\
-4 \\
-3 \\
-3 \\
-2 \\
-3 \\
-3 \\
-3
\end{array}\right),
$$

in the $\overline{M S}$ scheme at $\mu=1 \mathrm{TeV}$. The corresponding correlation matrix is given in appendix A. The mixing between operators produces larger diagonal errors for the pseudoscalar WC and induce very large non-diagonal entries. As explained before such large correlations reflect the fact that certain WC combination are much more constrained than the individual couplings.

With the values of the $\epsilon_{i}$ expressed at the high-energy scale, one can now translate them into determinations of the WC of the SMEFT, that we will denote as $\alpha_{i}$. The (tree-level) matching equations between the low-energy EFT and the SMEFT are [38]:

$$
\begin{aligned}
\frac{\delta G_{F}}{G_{F}} & =2\left[\hat{\alpha}_{\varphi l}^{(3)}\right]_{11+22}-\left[\hat{\alpha}_{l l}^{(1)}\right]_{1221}-2\left[\hat{\alpha}_{l l}^{(3)}\right]_{1122-\frac{1}{2}(1221)}, \\
V_{1 j} \cdot \epsilon_{L}^{j \ell} & =2 V_{1 j}\left[\hat{\alpha}_{\varphi l}^{(3)}\right]_{\ell \ell}+2\left[V \hat{\alpha}_{\varphi q}^{(3)}\right]_{1 j}-2\left[V \hat{\alpha}_{l q}^{(3)}\right]_{\ell \ell 1 j}, \\
V_{1 j} \cdot \epsilon_{R}^{j} & =-\left[\hat{\alpha}_{\varphi \varphi}\right]_{1 j} \\
V_{1 j} \cdot \epsilon_{s_{L}}^{j \ell} & =-\left[\hat{\alpha}_{l q}\right]_{\ell \ell j 1}^{*} \\
V_{1 j} \cdot \epsilon_{s_{R}}^{j \ell} & =-\left[V \hat{\alpha}_{q d e}^{\dagger}\right]_{\ell \ell 1 j} \\
V_{1 j} \cdot \epsilon_{T}^{j \ell} & =-\left[\hat{\alpha}_{l q}^{t}\right]_{\ell \ell j 1}^{*},
\end{aligned}
$$

where we labeled the quark generations with numbers, and introduced, for simplicity, $\epsilon_{s_{L / R}}^{j \ell}=\left(\epsilon_{S}^{j \ell} \pm \epsilon_{P}^{j \ell}\right) / 2$. In eqs. (6.4) the repeated indices $j, \ell$ are not summed over, while the index $m$ is. Finally let us also notice that $2 \hat{\alpha}_{i}=\alpha_{i} v^{2} / \Lambda^{2}$, with $v=\left(\sqrt{2} G_{F}\right)^{-1 / 2} \simeq$ $246 \mathrm{GeV}$.

The matching equation for $\epsilon_{R}^{D}$ shows clearly that this WC is lepton independent, cf. eq. (2.3), at this order in the SMEFT expansion, since the corresponding operator is $\mathcal{O}_{\varphi \varphi}=$ $i\left(\varphi^{T} \epsilon D_{\mu} \varphi\right)\left(\bar{u} \gamma^{\mu} d\right)+$ h.c.

Concerning the complementarity with collider searches, it is useful to notice the difference between chirality-conserving and -violating operators. On one hand, we have the SMEFT Wilson Coefficients contributing to $\delta G_{F}$ and $\epsilon_{L}^{j \ell}$, and probed in our fit through 
$\Delta_{\mathrm{CKM}}, \Delta_{L P}^{d}$ and $\Delta_{L}^{s}$, which conserve chirality. Their interference with the SM is not suppressed in collider observables and an interesting interplay with LEP [114, 115] and LHC $[47,116,117]$ searches is expected.

On the other hand, the SMEFT Wilson Coefficients contributing to $\epsilon_{R}^{d}, \epsilon_{S}^{s \ell}, \epsilon_{P}^{s \ell}$ and $\epsilon_{T}^{s \ell}$ are chirality-flipping, and thus they are not accessible by LEP searches at order $v^{2} / \Lambda^{2}$. In the case of (pseudo)scalar and tensor operators, the LHC can still provide interesting limits, thanks to the $\mathcal{O}\left(s^{2} / v^{4}\right)$ enhancement of the quadratic term (due to their contact-interaction nature) $[47,116]$, as we will discuss in section 6.3 . However, for $\epsilon_{R}^{d}$ this is not the case, as it is generated by non-standard $W$ couplings to right-handed quarks. The (semi)leptonic decays studied in this paper provide clearly a unique probe for these operators without competitors in the collider frontier.

Finally, in the matching equations of eq. (6.4) we used the basis of operators employed in ref. [38], which was a modified version of the seminal Buchmuller-Wyler basis [42] with the relevant redundancies (and the addition of one missing operator) properly taken care of. $^{8}$ Additional redundancies in other sectors of the Buchmuller-Wyler basis (not relevant for semileptonic quark decays) were later identified in ref. [113], where the first minimal and complete SMEFT basis was derived. In the sector relevant for our work, this so-called Warsaw basis is in fact very similar to the one of ref. [38] used in this work, up to some numerical factors and conventions. In particular, in these bases the equations of motions were not used to remove any operator containing fermions in favor of purely fermionic operators, which would introduced some complications in our flavor general analysis.

\subsection{MFV limit}

Once again it is interesting to have a look at the $\mathrm{U}(3)^{5}$-symmetric limit, which is in practice equivalent to the MFV case. In that limit we recover the result obtained in ref. [38], namely

$$
\Delta_{\mathrm{CKM}}=2 \frac{v^{2}}{\Lambda^{2}}\left(-\alpha_{\varphi l}^{(3)}+\alpha_{\varphi q}^{(3)}-\alpha_{\ell q}^{(3)}+\alpha_{l l}^{(3)}\right) .
$$

The bound on this combination of WC from (semi)leptonic hadron decays, $\Delta_{\mathrm{CKM}}=-(4.6 \pm$ $5.2) \times 10^{-4}$, cf. eq. (5.11), corresponds to an effective scale $\Lambda>10 \mathrm{TeV}(90 \% \mathrm{CL})$. As shown in ref. [38], such bound is much stronger than the limit obtained from the combined analysis of LEP and other EW precision observables. Thus, it is an important input for global EFT fits performed in this limit $[118,119] .{ }^{9}$ Finally let us notice that this is even more the case if a non-linear EFT framework is used, since more operators have to be considered [120].

\subsection{Bounds on scalar and tensor interactions}

If the new particles are too heavy to be produced on-shell at the LHC we can connect collider searches with low-energy processes in an elegant model-independent way using the

\footnotetext{
${ }^{8}$ One redundancy was not eliminated on purpose (either $O_{l l}^{(3)}$ or $O_{l l}^{(1)}$ ), so that each operator is proportional to the unit matrix in the $\mathrm{U}(3)^{5}$-symmetric case [38]. The Warsaw basis [113] chooses instead to keep only the operator $O_{l l}^{(1)}$, which in the $\mathrm{U}(3)^{5}$-symmetric limit has two independent flavor contractions.

${ }^{9}$ Ref. [119] does not work in the $\mathrm{U}(3)^{5}$-symmetric case, but in a more restrictive scenario, since the two independent contractions of flavor indices allowed by the $\mathrm{U}(3)^{5}$ symmetry for the operator $Q_{\ell \ell}$ (corresponding to $\alpha_{l l}^{(1)}$ and $\alpha_{l l}^{(3)}$ in our basis) are controlled by one single coefficients $C_{\ell \ell}$ in that work.
} 
SMEFT $[47,116]$. Since we are interested in non-standard effects in semileptonic $D$-quark decays, $D \rightarrow u \ell \bar{\nu}$, the natural channel to study at the LHC is $p p \rightarrow \ell+\mathrm{MET}+X$, since this process is sensitive at tree level to non-standard $\bar{u} D \rightarrow \ell \bar{\nu}$ partonic interactions. The comparative analysis between the bounds from nuclear $\beta$ decays and the LHC for WC involving the $d$-quark was performed in refs. $[47,116]$. The study was extended to the hyperon $\beta$ decays for the operators involving the $s$-quark in ref. [76], and we extend it further here by comparing the LHC bounds and those obtained for $\epsilon_{S, T}^{s \ell}$ from $K_{\ell 3}$.

First we briefly explain how the LHC bounds were obtained in refs. $[47,76,116]$. By using the matching relations in eqs. (6.4), one can express the cross-section $\sigma(p p \rightarrow$ $\ell+\mathrm{MET}+X)$ as is modified by non-standard $\bar{u} s \rightarrow \ell \bar{\nu}$ partonic interactions:

$$
\sigma\left(m_{T}>\bar{m}_{T}\right)=\sigma_{W}+\sigma_{S}\left|\epsilon_{S}^{s \ell}\right|^{2}+\sigma_{T}\left|\epsilon_{T}^{s \ell}\right|^{2},
$$

where $\sigma_{W}\left(\bar{m}_{T}\right)$ represents the SM contribution and $\sigma_{S, T}\left(\bar{m}_{T}\right)$ are new functions, with transverse mass higher than $\bar{m}_{T}$, which explicit form can be found in ref. [116]. The crucial feature is that they are several orders of magnitudes larger than the SM contribution, what compensates for the smallness of the NP couplings and makes possible to put significant bounds on them from these searches. Thus, comparing the observed events above $\bar{m}_{T}$ with the SM expectation we can set bounds on $\epsilon_{S, T}^{s \ell}$.

Some caveats are in order. First, it is important to note that the dependence of the cross section (6.6) on the WC is quadratic. We assume that contributions from SMEFT dimension- 8 operators can be neglected, which is expected to happen for a broad class of NP models (see e.g. ref. [121]). And secondly, this cross section is sensitive to a plethora of other dimension-6 effective operators, some of them interfering with the SM, which make possible the appearance of flat directions that we neglect here. It is worth stressing that these assumptions were not necessary in the low-energy fit.

Using $20 \mathrm{fb}^{-1}$ of data recorded at $\sqrt{s}=8 \mathrm{TeV}$ by the CMS collaboration in the electron channel $p p \rightarrow e^{ \pm}+$MET $+X$ [122], and choosing $\bar{m}_{T}=1.5 \mathrm{TeV}$, the $90 \%$ C.L. limit shown in figure 4 (left panel) was obtained in ref. [76]. In particular, one event is found with a transverse mass above $\bar{m}_{T}=1.5 \mathrm{TeV}$ in the $20 \mathrm{fb}^{-1}$ dataset recorded at $\sqrt{s}=8 \mathrm{TeV}$ by the CMS collaboration [122], in good agreement with the SM background of $2.02 \pm 0.26$ events. We repeat the same analysis here for the muonic channel $p p \rightarrow \mu^{ \pm}+\mathrm{MET}+X$ [122], where good agreement is also observed between data $\left(3\right.$ events above $\left.\bar{m}_{T}=1.5 \mathrm{TeV}\right)$ and $\mathrm{SM}(2.35 \pm 0.70$ events), obtaining the bound shown in figure 4 (right panel). The terms $\sigma_{S, T}$ were calculated using the MSTW2008 PDF sets evaluated at $Q^{2}=1 \mathrm{TeV}^{2}$ [123]. Further details can be found in ref. [116]. The running of the limits from $1 \mathrm{TeV}$ to $2 \mathrm{GeV}$ is performed using the QCD+EW RGE, cf. eq. (6.2).

The figure shows also the limits obtained in ref. [76] from the $R_{B_{1} B_{2}}$ ratio in eq. (3.26) and the existing data in several semileptonic hyperon decays, and the limits obtained in this work from the global analysis of $D \rightarrow u \ell \bar{\nu}$ processes.

Figure 4 illustrates the interesting complementarity between low-energy experiments and the LHC searches. While the LHC cross section is almost equally sensitive to both electron and muon couplings, $K_{\ell 3(\gamma)}$ is much more sensitive to the muon one, where the interference with the SM is the dominant contribution. The figure illustrates quite clearly 

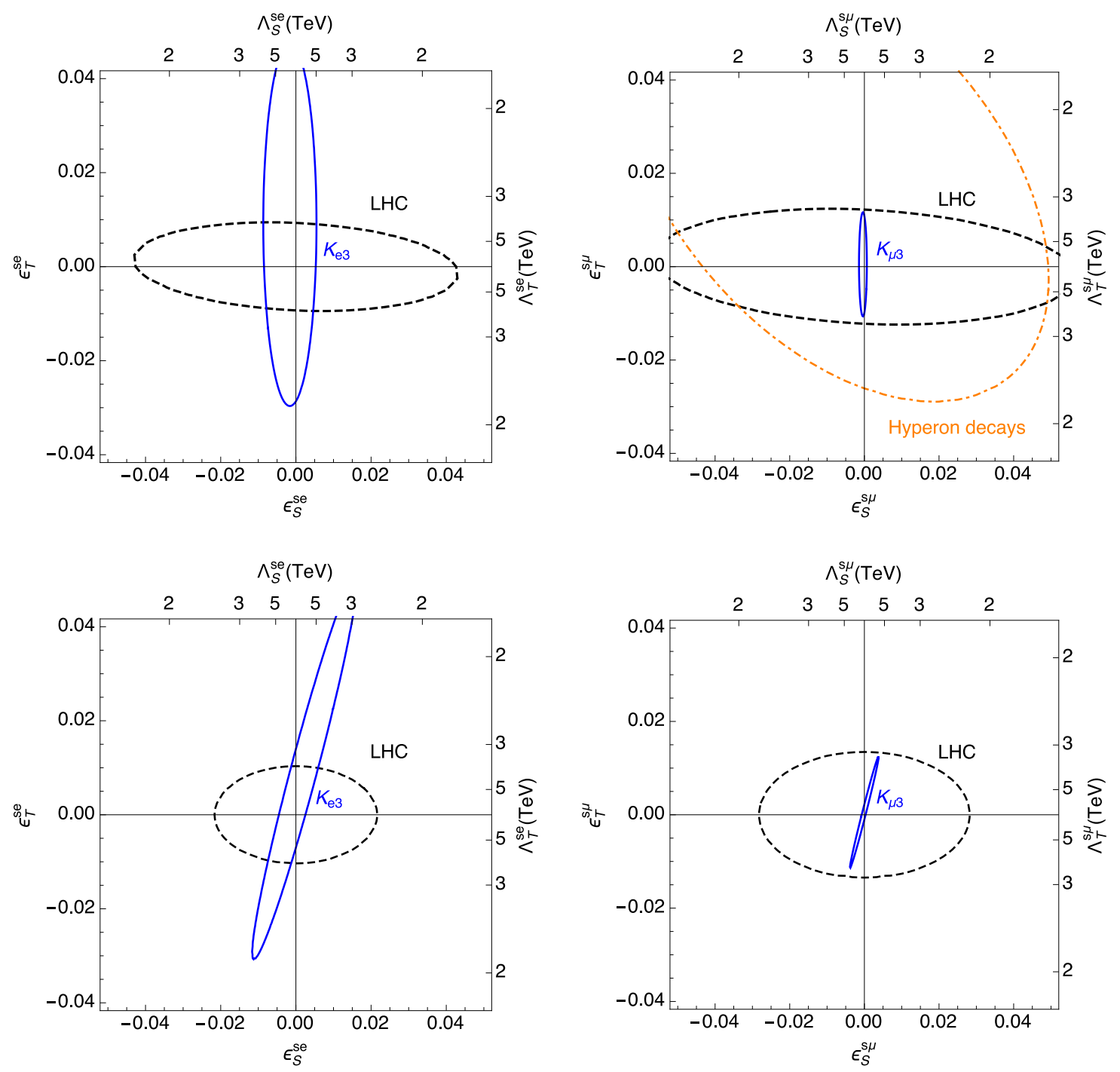

Figure 4. $90 \%$ CL constraints on $\epsilon_{S, T}^{s \ell}$ from our global fit (blue solid ellipse), from the analysis of $p p \rightarrow \ell^{ \pm}+\mathrm{MET}+X$ CMS data (black dashed ellipse) and from semileptonic hyperon decays (orange dot-dashed lines) [76]. The left (right) panel corresponds to the electronic (muonic) couplings, whereas the upper (lower) panel show the results at $\mu=2 \mathrm{GeV}(1 \mathrm{TeV})$. Effective scales are defined by $\Lambda_{i} \approx\left(V_{u s} \epsilon_{i}\right)^{-1 / 2} v$, see eqs. (6.4).

that semileptonic kaon decays are exploring new regions in the NP parameter space unaccessible for the LHC and corresponding to 1-10 TeV effective scales. Finally, the lower panel of figure 4 shows the same limits, this time at $\mu=1 \mathrm{TeV}$, which might be more interesting from a model-building perspective.

Needless to say, this interplay becomes much more interesting if a discrepancy with the $\mathrm{SM}$ is found. This was illustrated in ref. [47] assuming the presence of a scalar resonance at the LHC that, in our case, could be detected as a nonzero $\epsilon_{S}^{s \ell}$ in $K_{\ell 3}$ (or a non-zero pseudoscalar/tensor coupling due to the RGE mixing). 


\section{Conclusions}

In this paper we introduce a global model-independent analysis of NP in the $D \rightarrow u \ell \nu$ transitions $(D=d, s ; \ell=e, \mu)$ in the context of the SMEFT. We do not assume any flavor symmetry and we keep all possible NP operators at the same time. Special attention is paid to the (semi)leptonic kaon decays where such a comprehensive and systematic analysis of NP was lacking, and we study the complementarity with pion decays and nuclear, neutron and hyperon $\beta$ decays. The latter become necessary since one can not discriminate among all different possible NP effects using only pion and kaon decay observables. This is not only relevant for the determination of $\left|\tilde{V}_{u d}^{e}\right|$ but also for singling out the effects of NP contributions from right-handed currents. In this sense, future analyses would greatly benefit from a better understanding of neutron and hyperon properties such as $g_{A}$ and $g_{1}$.

Besides providing a road map for future tests of the SM using all these processes, we provide numerical results of a fit using current experimental data and lattice QCD results. Our analysis includes the MFV and the SM limit as a specific case. In fact our output are not only the bounds on the various WC, but also the $V_{u s}$ and $V_{u d}$ elements, and includes various QCD form factors parameters. In the SM limit we recover the most precise determinations of them, with small improvements due to the inclusion of the individual rate of $K_{\mu 2}$ as a separate input and the Callan-Treiman theorem.

We find that these decays are sensitive to NP with typical scales of several $\mathrm{TeV}$, especially in the case of a pseudoscalar contribution to $P_{e 2}^{ \pm}(P=K, \pi)$, which is ruled out up to scales as high as $\mathcal{O}(100) \mathrm{TeV}$. To make this connection to the high-energy scale more explicit, we properly accounted for the operator running and mixing under the QCD and EW interactions, we expressed the low-energy bounds in the context of the SMEFT and provided their value at $1 \mathrm{TeV}$. Our results can then be matched straightforwardly to any specific NP model. Very large correlations appear between different WC, which should have non-trivial implications for the chosen model. Finally, as illustrated with a few simple cases in this work, the matching with the SMEFT opens the possibility for powerful synergies between these decays and searches of NP at the LHC.

\section{Acknowledgments}

The authors thank L. Alvarez-Ruso, V. Cirigliano, J. C. Hardy, V. Lubicz, M. Moulson, A. Pich, J. Portolés, I. C. Towner, M. Vicente Vacas, F. Wauters, and O. Yushchenko for useful discussions and correspondence. We would also like to acknowledge the Institute for Nuclear Theory (INT) and the Mainz Institute for Theoretical Physics (MITP) for enabling us to complete a significant portion of this work. M.G.-A. is grateful to the LABEX Lyon Institute of Origins (ANR-10-LABX-0066) of the Université de Lyon for its financial support within the program ANR-11-IDEX-0007 of the French government. JMC's work is funded by the People Programme (Marie Curie Actions) of the European Union's Seventh Framework Programme (FP7/2007-2013) under REA grant agreement n PIOF-GA-2012-330458. 


\section{A WC bounds at $\mu=1 \mathrm{TeV}$}

$$
\left(\begin{array}{c}
\tilde{V}_{u d}^{e} \\
\Delta_{\mathrm{CKM}} \\
\Delta_{L}^{s} \\
\epsilon_{L}^{d e}-\epsilon_{L}^{d \mu}+0.22 \epsilon_{S}^{d \mu}+47 \epsilon_{P}^{d \mu}-15 \epsilon_{T}^{d \mu} \\
\epsilon_{R}^{d} \\
\epsilon_{R}^{s} \\
\epsilon_{S}^{d e} \\
\epsilon_{P}^{d e} \\
\epsilon_{T}^{d e} \\
\epsilon_{S}^{s e} \\
\epsilon_{P}^{s e} \\
\epsilon_{T}^{s e} \\
\epsilon_{S}^{s \mu} \\
\epsilon_{P}^{s \mu} \\
\epsilon_{T}^{s \mu}
\end{array}\right)=\left(\begin{array}{c}
0.97451 \pm 0.00038 \\
-1.2 \pm 8.4 \\
1.0 \pm 2.5 \\
1.9 \pm 3.8 \\
-1.3 \pm 1.7 \\
0.1 \pm 5.0 \\
7.4 \pm 7.1 \\
0.3 \pm 2.8 \\
1.1 \pm 8.7 \\
2.3 \pm 6.5 \\
3.1 \pm 6.2 \\
1.0 \pm 1.9 \\
0.0 \pm 1.8 \\
-0.2 \pm 2.9 \\
0.6 \pm 5.6
\end{array}\right) \times 10^{-1} \quad\left(\begin{array}{c}
0 \\
-4 \\
-3 \\
-2 \\
-2 \\
-2 \\
-4 \\
-4 \\
-3 \\
-3 \\
-2 \\
-3 \\
-3 \\
-3
\end{array}\right),
$$

in the $\overline{M S}$ scheme at $\mu=1 \mathrm{TeV}$. Let us remind the reader that $\Delta_{L}^{s}=\epsilon_{L}^{s \mu}-\epsilon_{L}^{s e}$. The correlation matrix is given by:

$$
\rho=\left(\begin{array}{ccccccccccccccc}
1.0 .88 & 0 . & 0.01 & 0 . & 0 . & 0.76 & -0.01 & 0 . & 0 . & 0 . & 0 . & 0 . & 0 . & 0 . \\
- & 1 . & -0.07 & 0.01 & 0 . & 0 . & 0.67 & 0 . & 0 . & 0 . & 0 . & 0 . & -0.02 & 0.01 & 0 . \\
- & - & 1 . & 0 . & 0 . & 0 . & 0 . & 0 . & 0 . & 0 . & 0 . & 0 . & 0.45 & 0.3 & 0.46 \\
- & - & - & 1 . & -0.87 & 0.0 .01 & 0.01 & 0 . & 0 . & 0 . & 0 . & 0.01 & 0.07 & 0 . \\
- & - & - & - & 1 . & 0 . & 0 . & -0.01 & 0 . & 0 . & 0 . & 0 . & 0 . & 0 . & 0 . \\
- & - & - & - & - & 1 . & 0 . & 0 . & 0 . & 0 . & 0 . & 0 . & 0 . & -0.75 & 0 . \\
- & - & - & - & - & - & 1 . & 0.38 & 0.39 & 0 . & 0 . & 0 . & 0 . & 0 . & 0 . \\
- & - & - & - & - & - & - & 1 . & 0.9998 & 0 . & 0 . & 0 . & 0 . & 0 . & 0 . \\
- & - & - & - & - & - & - & - & 1 . & 0 . & 0 . & 0 . & 0 . & 0 . & 0 . \\
- & - & - & - & - & - & - & - & - & 1.0 .96 & 0.97 & 0 . & 0 . & 0 . \\
- & - & - & - & - & - & - & - & - & - & 1 . & 0.999998 & 0 . & 0 . & 0 . \\
- & - & - & - & - & - & - & - & - & - & - & 1 . & 0 . & 0 . & 0 . \\
- & - & - & - & - & - & - & - & - & - & - & - & 1 . & 0.63 & 0.99 \\
- & - & - & - & - & - & - & - & - & - & - & - & - & 1 . & 0.64 \\
- & - & - & - & - & - & - & - & - & - & - & - & - & - & 1 .
\end{array}\right) .
$$

Open Access. This article is distributed under the terms of the Creative Commons Attribution License (CC-BY 4.0), which permits any use, distribution and reproduction in any medium, provided the original author(s) and source are credited.

\section{References}

[1] Flavianet Working Group on Kaon Decays collaboration, M. Antonelli et al., Precision tests of the standard model with leptonic and semileptonic kaon decays, arXiv:0801.1817 [INSPIRE]. 
[2] Flavianet Working Group on Kaon Decays collaboration, M. Antonelli et al., An evaluation of $\left|V_{u s}\right|$ and precise tests of the Standard Model from world data on leptonic and semileptonic kaon decays, Eur. Phys. J. C 69 (2010) 399 [arXiv:1005.2323] [InSPIRE].

[3] V. Cirigliano, G. Ecker, H. Neufeld, A. Pich and J. Portoles, Kaon decays in the standard model, Rev. Mod. Phys. 84 (2012) 399 [arXiv:1107.6001] [INSPIRE].

[4] Particle Data Group collaboration, K.A. Olive et al., Review of particle physics, Chin. Phys. C 38 (2014) 090001 [INSPIRE].

[5] S. Aoki et al., Review of lattice results concerning low-energy particle physics, Eur. Phys. J. C 74 (2014) 2890 [arXiv:1310.8555] [INSPIRE].

[6] J.L. Rosner, S. Stone and R.S. Van de Water, Leptonic Decays of Charged Pseudoscalar Mesons - 2015, submitted to Particle Data Book (2015), arXiv:1509.02220 [INSPIRE].

[7] J. Gasser and H. Leutwyler, Chiral perturbation theory: expansions in the mass of the strange quark, Nucl. Phys. B 250 (1985) 465 [INSPIRE].

[8] J. Gasser and H. Leutwyler, Low-energy expansion of meson form-factors, Nucl. Phys. B 250 (1985) 517 [INSPIRE].

[9] V. Cirigliano, M. Knecht, H. Neufeld, H. Rupertsberger and P. Talavera, Radiative corrections to K(l3) decays, Eur. Phys. J. C 23 (2002) 121 [hep-ph/0110153] [INSPIRE].

[10] J. Bijnens and P. Talavera, K(l3) decays in chiral perturbation theory, Nucl. Phys. B 669 (2003) 341 [hep-ph/0303103] [INSPIRE].

[11] M. Jamin, J.A. Oller and A. Pich, Strangeness changing scalar form-factors, Nucl. Phys. B 622 (2002) 279 [hep-ph/0110193] [rNSPIRE].

[12] B. Ananthanarayan and B. Moussallam, Four-point correlator constraints on electromagnetic chiral parameters and resonance effective Lagrangians, JHEP 06 (2004) 047 [hep-ph/0405206] [INSPIRE].

[13] M. Jamin, J.A. Oller and A. Pich, Order $p^{6}$ chiral couplings from the scalar K $\pi$ form-factor, JHEP 02 (2004) 047 [hep-ph/0401080] [INSPIRE].

[14] V. Cirigliano, G. Ecker, M. Eidemuller, R. Kaiser, A. Pich and J. Portoles, The $\langle S P P>$ Green function and $\mathrm{SU}(3)$ breaking in $K(l 3)$ decays, JHEP 04 (2005) 006 [hep-ph/0503108] [INSPIRE].

[15] S. Descotes-Genon and B. Moussallam, Radiative corrections in weak semi-leptonic processes at low energy: a two-step matching determination, Eur. Phys. J. C 42 (2005) 403 [hep-ph/0505077] [INSPIRE].

[16] V. Cirigliano and I. Rosell, Two-loop effective theory analysis of $\pi(K) \rightarrow e \bar{\nu}_{e} \gamma$ branching ratios, Phys. Rev. Lett. 99 (2007) 231801 [arXiv:0707.3439] [INSPIRE].

[17] V. Cirigliano and I. Rosell, $\pi / K-\rightarrow e \bar{n} \bar{u}_{e}$ branching ratios to $O\left(e^{2} p^{4}\right)$ in chiral perturbation theory, JHEP 10 (2007) 005 [arXiv:0707.4464] [INSPIRE].

[18] A. Kastner and H. Neufeld, The K(l3) scalar form factors in the standard model, Eur. Phys. J. C 57 (2008) 541 [arXiv:0805.2222] [inSPIRE].

[19] V. Cirigliano, M. Giannotti and H. Neufeld, Electromagnetic effects in K(l3) decays, JHEP 11 (2008) 006 [arXiv:0807.4507] [INSPIRE]. 
[20] V. Bernard, M. Oertel, E. Passemar and J. Stern, Dispersive representation and shape of the K(l3) form factors: robustness, Phys. Rev. D 80 (2009) 034034 [arXiv:0903.1654] [INSPIRE].

[21] V. Cirigliano and H. Neufeld, A note on isospin violation in Pl2 $(\gamma)$ decays, Phys. Lett. B 700 (2011) 7 [arXiv:1102.0563] [INSPIRE].

[22] BABAR collaboration, J.P. Lees et al., Evidence for an excess of $\bar{B} \rightarrow D^{(*)} \tau^{-} \bar{\nu}_{\tau}$ decays, Phys. Rev. Lett. 109 (2012) 101802 [arXiv:1205.5442] [InSPIRE].

[23] BABAR collaboration, J.P. Lees et al., Measurement of an excess of $\bar{B} \rightarrow D^{(*)} \tau^{-} \bar{\nu}_{\tau}$ decays and implications for charged Higgs bosons, Phys. Rev. D 88 (2013) 072012 [arXiv:1303.0571] [INSPIRE].

[24] LHCb collaboration, Test of lepton universality using $B^{+} \rightarrow K^{+} \ell^{+} \ell^{-}$decays, Phys. Rev. Lett. 113 (2014) 151601 [arXiv: 1406.6482] [INSPIRE].

[25] LHCb collaboration, Measurement of the ratio of branching fractions $\mathcal{B}\left(\bar{B}^{0} \rightarrow D^{*+} \tau^{-} \bar{\nu}_{\tau}\right) / \mathcal{B}\left(\bar{B}^{0} \rightarrow D^{*+} \mu^{-} \bar{\nu}_{\mu}\right)$, Phys. Rev. Lett. 115 (2015) 111803 [arXiv: 1506.08614] [INSPIRE].

[26] BeLLE collaboration, M. Huschle et al., Measurement of the branching ratio of $\bar{B} \rightarrow D^{(*)} \tau^{-} \bar{\nu}_{\tau}$ relative to $\bar{B} \rightarrow D^{(*)} \ell^{-} \bar{\nu}_{\ell}$ decays with hadronic tagging at Belle, Phys. Rev. D 92 (2015) 072014 [arXiv: 1507.03233] [INSPIRE].

[27] LHCb collaboration, Angular analysis of the $B^{0} \rightarrow K^{* 0} \mu^{+} \mu^{-}$decay using $3 f^{-1}$ of integrated luminosity, JHEP 02 (2016) 104 [arXiv:1512.04442] [INSPIRE].

[28] Belle collaboration, A. Abdesselam et al., Measurement of the branching ratio of $\bar{B}^{0} \rightarrow D^{*+} \tau^{-} \bar{\nu}_{\tau}$ relative to $\bar{B}^{0} \rightarrow D^{*+} \ell^{-} \bar{\nu}_{\ell}$ decays with a semileptonic tagging method, arXiv:1603.06711 [INSPIRE].

[29] Belle collaboration, A. Abdesselam et al., Angular analysis of $B^{0} \rightarrow K^{*}(892)^{0} \ell^{+} \ell^{-}$, arXiv: 1604.04042 [INSPIRE].

[30] A.J. Buras, M. Gorbahn, S. Jäger and M. Jamin, Improved anatomy of $\epsilon^{\prime} / \epsilon$ in the standard model, JHEP 11 (2015) 202 [arXiv:1507.06345] [INSPIRE].

[31] A. Masiero, P. Paradisi and R. Petronzio, Probing new physics through $\mu$-e universality in $K \rightarrow l \nu$, Phys. Rev. D 74 (2006) 011701 [hep-ph/0511289] [INSPIRE].

[32] V. Bernard, M. Oertel, E. Passemar and J. Stern, $K(\mu 3)^{L}$ decay: a stringent test of right-handed quark currents, Phys. Lett. B 638 (2006) 480 [hep-ph/0603202] [INSPIRE].

[33] V. Bernard, M. Oertel, E. Passemar and J. Stern, Tests of non-standard electroweak couplings of right-handed quarks, JHEP 01 (2008) 015 [arXiv:0707.4194] [INSPIRE].

[34] B.A. Campbell and A. Ismail, Leptonic pion decay and physics beyond the electroweak standard model, arXiv:0810.4918 [INSPIRE].

[35] M. Carpentier and S. Davidson, Constraints on two-lepton, two quark operators, Eur. Phys. J. C 70 (2010) 1071 [arXiv: 1008.0280] [InSPIRE].

[36] M. Jung, A. Pich and P. Tuzon, Charged-Higgs phenomenology in the aligned two-Higgs-doublet model, JHEP 11 (2010) 003 [arXiv:1006.0470] [INSPIRE].

[37] S. Bauman, J. Erler and M. Ramsey-Musolf, Charged current universality and the MSSM, Phys. Rev. D 87 (2013) 035012 [arXiv:1204.0035] [INSPIRE]. 
[38] V. Cirigliano, J. Jenkins and M. Gonzalez-Alonso, Semileptonic decays of light quarks beyond the Standard Model, Nucl. Phys. B 830 (2010) 95 [arXiv:0908.1754] [INSPIRE].

[39] N. Severijns, M. Beck and O. Naviliat-Cuncic, Tests of the standard electroweak model in $\beta$ decay, Rev. Mod. Phys. 78 (2006) 991 [nucl-ex/0605029] [INSPIRE].

[40] F. Wauters, A. García and R. Hong, Limits on tensor-type weak currents from nuclear and neutron $\beta$ decays, Phys. Rev. C 89 (2014) 025501 [arXiv: 1306.2608] [INSPIRE].

[41] R.W. Pattie, K.P. Hickerson and A.R. Young, Limits on tensor coupling from neutron $\beta$-decay, Phys. Rev. C 88 (2013) 048501 [arXiv:1309.2499] [inSPIRE].

[42] W. Buchmüller and D. Wyler, Effective lagrangian analysis of new interactions and flavor conservation, Nucl. Phys. B 268 (1986) 621 [INSPIRE].

[43] M. Moulson, Experimental determination of $V_{u s}$ from kaon decays, talk given at $8^{\text {th }}$ International Workshop on the CKM Unitarity Triangle (CKM2014), September 8-12, Vienna, Austria (2014), arXiv:1411.5252 [INSPIRE].

[44] R. Alonso, B. Grinstein and J. Martin Camalich, $\mathrm{SU}(2) \times U(1)$ gauge invariance and the shape of new physics in rare B decays, Phys. Rev. Lett. 113 (2014) 241802 [arXiv: 1407.7044] [INSPIRE].

[45] J. Aebischer, A. Crivellin, M. Fael and C. Greub, Matching of gauge invariant dimension-six operators for $b \rightarrow s$ and $b \rightarrow c$ transitions, JHEP 05 (2016) 037 [arXiv: 1512.02830] [INSPIRE].

[46] R. Alonso, B. Grinstein and J. Martin Camalich, Lepton universality violation and lepton flavor conservation in B-meson decays, JHEP 10 (2015) 184 [arXiv: 1505.05164] [INSPIRE].

[47] T. Bhattacharya et al., Probing novel scalar and tensor interactions from (ultra)cold neutrons to the LHC, Phys. Rev. D 85 (2012) 054512 [arXiv:1110.6448] [INSPIRE].

[48] A. Sirlin, Large $m(W), m(Z)$ behavior of the $O(\alpha)$ corrections to semileptonic processes mediated by W, Nucl. Phys. B 196 (1982) 83 [inSPIRE].

[49] W.J. Marciano and A. Sirlin, Radiative corrections to $\pi(l 2)$ decays, Phys. Rev. Lett. 71 (1993) 3629 [INSPIRE].

[50] M.B. Voloshin, Upper bound on tensor interaction in the decay $\pi^{-} \rightarrow e^{-} \bar{\nu} \gamma$, Phys. Lett. B 283 (1992) 120 [INSPIRE].

[51] P. Herczeg, On the question of a tensor interaction in $\pi \rightarrow e$ electron-neutrino $\gamma$ decay, Phys. Rev. D 49 (1994) 247 [inSPIRE].

[52] B.A. Campbell and D.W. Maybury, Constraints on scalar couplings from $\pi^{ \pm} \rightarrow l^{ \pm} \nu(l)$, Nucl. Phys. B 709 (2005) 419 [hep-ph/0303046] [INSPIRE].

[53] R. Alonso, E.E. Jenkins, A.V. Manohar and M. Trott, Renormalization group evolution of the standard model dimension six operators III: gauge coupling dependence and phenomenology, JHEP 04 (2014) 159 [arXiv: 1312.2014] [INSPIRE].

[54] M. Gonzalez-Alonso, A. Greljo, G. Isidori and D. Marzocca, Pseudo-observables in Higgs decays, Eur. Phys. J. C 75 (2015) 128 [arXiv:1412.6038] [INSPIRE].

[55] M. Finkemeier, Radiative corrections to $\pi(l 2)$ and $K(l 2)$ decays, Phys. Lett. B 387 (1996) 391 [hep-ph/9505434] [INSPIRE]. 
[56] V.N. Bolotov et al., The experimental study of the $\pi^{-} \rightarrow e^{-} \bar{\nu} \gamma$ decay in flight, Phys. Lett. B 243 (1990) 308 [INSPIRE].

[57] M. Bychkov et al., New precise measurement of the pion weak form factors in $\pi^{+} \rightarrow e^{+} \nu \gamma$ decay, Phys. Rev. Lett. 103 (2009) 051802 [arXiv:0804.1815] [INSPIRE].

[58] KLOE collaboration, F. Ambrosino et al., Precise measurement of $\Gamma(K \rightarrow e \nu(\gamma)) / \Gamma(K \rightarrow \mu \nu(\gamma))$ and study of $K \rightarrow e \nu \gamma$, Eur. Phys. J. C 64 (2009) 627 [Erratum ibid. 65 (2010) 703] [arXiv:0907.3594] [INSPIRE].

[59] J. Bijnens, G. Ecker and J. Gasser, Radiative semileptonic kaon decays, Nucl. Phys. B 396 (1993) 81 [hep-ph/9209261] [INSPIRE].

[60] A.A. Poblaguev, On the $\pi \rightarrow$ e neutrino $\gamma$ decay sensitivity to a tensor coupling in the effective quark lepton interaction, Phys. Lett. B 238 (1990) 108 [INSPIRE].

[61] M.V. Chizhov, Search for tensor interactions in kaon decays at DAPHNE, Phys. Lett. B 381 (1996) 359 [hep-ph/9511287] [INSPIRE].

[62] V. Mateu and J. Portoles, Form-factors in radiative pion decay, Eur. Phys. J. C 52 (2007) 325 [arXiv: 0706.1039] [INSPIRE].

[63] O.P. Yushchenko et al., High statistic study of the $K^{-} \rightarrow \pi^{0} \mu^{-} \nu$ decay, Phys. Lett. B 581 (2004) 31 [hep-ex/0312004] [INSPIRE].

[64] O.P. Yushchenko et al., High statistic measurement of the $\mathrm{K}^{-} \rightarrow \pi^{0} e^{-} \nu$ decay form-factors, Phys. Lett. B 589 (2004) 111 [hep-ex/0404030] [INSPIRE].

[65] NA48 collaboration, A. Lai et al., Measurement of $K^{0}(e 3)$ form-factors, Phys. Lett. B 604 (2004) 1 [hep-ex/0410065] [INSPIRE].

[66] KTEV collaboration, T. Alexopoulos et al., Measurements of semileptonic $K(L)$ decay form-factors, Phys. Rev. D 70 (2004) 092007 [hep-ex/0406003] [INSPIRE].

[67] KLOE collaboration, F. Ambrosino et al., Measurement of the form-factor slopes for the decay $K(L) \rightarrow \pi^{ \pm} e^{\mp} \nu$ with the KLOE detector, Phys. Lett. B 636 (2006) 166 [hep-ex/0601038] [INSPIRE].

[68] KLOE collaboration, F. Ambrosino et al., Measurement of the $K(L) \rightarrow \pi \mu \nu$ form-factor parameters with the KLOE detector, JHEP 12 (2007) 105 [arXiv:0710.4470] [INSPIRE].

[69] NA48 collaboration, A. Lai et al., Measurement of $K^{0}(\mu 3)$ form factors, Phys. Lett. B 647 (2007) 341 [hep-ex/0703002] [INSPIRE].

[70] KTEV collaboration, E. Abouzaid et al., Dispersive analysis of $K(l \mu 3)$ and $K(l e 3)$ scalar and vector form factors using KTeV data, Phys. Rev. D 81 (2010) 052001 [arXiv: 0912.1291] [INSPIRE].

[71] C.G. Callan and S.B. Treiman, Equal time commutators and K meson decays, Phys. Rev. Lett. 16 (1966) 153 [INSPIRE].

[72] J. Bijnens and K. Ghorbani, Isospin breaking in $K \pi$ vector form-factors for the weak and rare decays $K(l 3), K \rightarrow \pi \nu \bar{\nu}$ and $K \rightarrow \pi \ell^{+} \ell^{-}$, arXiv:0711.0148 [INSPIRE].

[73] N. Carrasco, P. Lami, V. Lubicz, L. Riggio, S. Simula and C. Tarantino, $K \rightarrow \pi$ semileptonic form factors with $N_{f}=2+1+1$ twisted mass fermions, Phys. Rev. D 93 (2016) 114512 [arXiv: 1602.04113] [INSPIRE]. 
[74] J.C. Hardy and I.S. Towner, Superallowed $0^{+} \rightarrow 0^{+}$nuclear $\beta$ decays: 2014 critical survey, with precise results for $V_{u d}$ and CKM unitarity, Phys. Rev. C 91 (2015) 025501 [arXiv:1411.5987] [INSPIRE].

[75] S. Weinberg, Charge symmetry of weak interactions, Phys. Rev. 112 (1958) 1375 [INSPIRE].

[76] H.-M. Chang, M. González-Alonso and J. Martin Camalich, Nonstandard semileptonic hyperon decays, Phys. Rev. Lett. 114 (2015) 161802 [arXiv:1412.8484] [INSPIRE].

[77] N. Cabibbo, E.C. Swallow and R. Winston, Semileptonic hyperon decays and CKM unitarity, Phys. Rev. Lett. 92 (2004) 251803 [hep-ph/0307214] [INSPIRE].

[78] V. Mateu and A. Pich, V(us) determination from hyperon semileptonic decays, JHEP 10 (2005) 041 [hep-ph/0509045] [INSPIRE].

[79] S. Sasaki, Hyperon vector form factor from $2+1$ flavor lattice QCD, Phys. Rev. D 86 (2012) 114502 [arXiv:1209.6115] [INSPIRE].

[80] L.-S. Geng, K.-W. Li and J. Martin Camalich, Chiral extrapolation and finite-volume dependence of the hyperon vector couplings, Phys. Rev. D 89 (2014) 113007 [arXiv: 1402.7133] [INSPIRE].

[81] P.E. Shanahan et al., SU(3) breaking in hyperon transition vector form factors, Phys. Rev. D 92 (2015) 074029 [arXiv: 1508.06923] [INSPIRE].

[82] D. Mund et al., Determination of the weak axial vector coupling from a measurement of the $\beta$-asymmetry parameter a in neutron $\beta$ decay, Phys. Rev. Lett. 110 (2013) 172502 [arXiv: 1204.0013] [INSPIRE].

[83] UCNA collaboration, M.P. Mendenhall et al., Precision measurement of the neutron $\beta$-decay asymmetry, Phys. Rev. C 87 (2013) 032501 [arXiv:1210.7048] [INSPIRE].

[84] J. Green, Hadron structure from lattice QCD, AIP Conf. Proc. 1701 (2016) 040007 [arXiv: 1412.4637] [INSPIRE].

[85] R. Horsley et al., Nucleon axial charge and pion decay constant from two-flavor lattice QCD, Phys. Lett. B 732 (2014) 41 [arXiv:1302.2233] [INSPIRE].

[86] G.S. Bali et al., Nucleon isovector couplings from $N_{f}=2$ lattice QCD, Phys. Rev. D 91 (2015) 054501 [arXiv:1412.7336] [INSPIRE].

[87] A. Abdel-Rehim et al., Nucleon and pion structure with lattice QCD simulations at physical value of the pion mass, Phys. Rev. D 92 (2015) 114513 [arXiv:1507.04936] [INSPIRE].

[88] N. Cabibbo, E.C. Swallow and R. Winston, Semileptonic hyperon decays, Ann. Rev. Nucl. Part. Sci. 53 (2003) 39 [hep-ph/0307298] [InSPIRE].

[89] M. González-Alonso and J. Martin Camalich, Isospin breaking in the nucleon mass and the sensitivity of $\beta$ decays to new physics, Phys. Rev. Lett. 112 (2014) 042501 [arXiv:1309.4434] [INSPIRE].

[90] O. Naviliat-Cuncic and M. González-Alonso, Prospects for precision measurements in nuclear $\beta$ decay at the LHC era, Annalen Phys. 525 (2013) 600 [arXiv:1304.1759] [INSPIRE].

[91] W.J. Marciano, Precise determination of $\left|V_{u s}\right|$ from lattice calculations of pseudoscalar decay constants, Phys. Rev. Lett. 93 (2004) 231803 [hep-ph/0402299] [INSPIRE]. 
[92] A. Abada, D. Das, A.M. Teixeira, A. Vicente and C. Weiland, Tree-level lepton universality violation in the presence of sterile neutrinos: impact for $R_{K}$ and $R_{\pi}$, JHEP 02 (2013) 048 [arXiv:1211.3052] [INSPIRE].

[93] A. de Gouvêa and A. Kobach, Global constraints on a heavy neutrino, Phys. Rev. D 93 (2016) 033005 [arXiv: 1511.00683] [inSPIRE].

[94] PIENu collaboration, A. Aguilar-Arevalo et al., Improved measurement of the $\pi \rightarrow e \nu$ branching ratio, Phys. Rev. Lett. 115 (2015) 071801 [arXiv: 1506. 05845] [INSPIRE].

[95] M. Moulson, private communication.

[96] I. Baum, V. Lubicz, G. Martinelli, L. Orifici and S. Simula, Matrix elements of the electromagnetic operator between kaon and pion states, Phys. Rev. D 84 (2011) 074503 [arXiv:1108.1021] [INSPIRE].

[97] J. Hardy and I. Towner, private communication.

[98] MILC collaboration, A. Bazavov et al., Nonperturbative QCD simulations with $2+1$ flavors of improved staggered quarks, Rev. Mod. Phys. 82 (2010) 1349 [arXiv:0903.3598] [INSPIRE].

[99] HPQCD, UKQCD collaboration, E. Follana, C.T.H. Davies, G.P. Lepage and J. Shigemitsu, High precision determination of the $\pi, K, D$ and $D(s)$ decay constants from lattice QCD, Phys. Rev. Lett. 100 (2008) 062002 [arXiv:0706.1726] [INSPIRE].

[100] S. Dürr et al., The ratio FK/Fpi in QCD, Phys. Rev. D 81 (2010) 054507 [arXiv: 1001.4692] [INSPIRE].

[101] RBC, UKQCD collaboration, R. Arthur et al., Domain wall QCD with near-physical pions, Phys. Rev. D 87 (2013) 094514 [arXiv:1208.4412] [INSPIRE].

[102] A. Bazavov et al., Kaon semileptonic vector form factor and determination of $\left|V_{u s}\right|$ using staggered fermions, Phys. Rev. D 87 (2013) 073012 [arXiv: 1212.4993] [INSPIRE].

[103] P.A. Boyle et al., The kaon semileptonic form factor with near physical domain wall quarks, JHEP 08 (2013) 132 [arXiv:1305.7217] [InSPIRE].

[104] H.-W. Lin and K. Orginos, First calculation of hyperon axial couplings from lattice QCD, Phys. Rev. D 79 (2009) 034507 [arXiv:0712.1214] [INSPIRE].

[105] QCDSF/UKQCD collaboration, M. Gockeler et al., Baryon axial charges and momentum fractions with $N_{f}=2+1$ dynamical fermions, PoS(LATTICE 2010)163 [arXiv:1102.3407] [INSPIRE].

[106] A.N. Cooke et al., SU(3) flavour breaking and baryon structure, PoS (LATTICE 2013) 278 [arXiv:1311.4916] [INSPIRE].

[107] T. Ledwig, J. Martin Camalich, L.S. Geng and M.J. Vicente Vacas, Octet-baryon axial-vector charges and $\mathrm{SU}(3)$-breaking effects in the semileptonic hyperon decays, Phys. Rev. D 90 (2014) 054502 [arXiv:1405.5456] [INSPIRE].

[108] R.S. Chivukula and H. Georgi, Composite technicolor standard model, Phys. Lett. B 188 (1987) 99 [InSPIRE].

[109] L.J. Hall and L. Randall, Weak scale effective supersymmetry, Phys. Rev. Lett. 65 (1990) 2939 [INSPIRE]. 
[110] A.J. Buras, P. Gambino, M. Gorbahn, S. Jager and L. Silvestrini, Universal unitarity triangle and physics beyond the standard model, Phys. Lett. B 500 (2001) 161 [hep-ph/0007085] [INSPIRE].

[111] G. D'Ambrosio, G.F. Giudice, G. Isidori and A. Strumia, Minimal flavor violation: an effective field theory approach, Nucl. Phys. B 645 (2002) 155 [hep-ph/0207036] [InSPIRE].

[112] V. Cirigliano, B. Grinstein, G. Isidori and M.B. Wise, Minimal flavor violation in the lepton sector, Nucl. Phys. B 728 (2005) 121 [hep-ph/0507001] [INSPIRE].

[113] B. Grzadkowski, M. Iskrzynski, M. Misiak and J. Rosiek, Dimension-six terms in the standard model lagrangian, JHEP 10 (2010) 085 [arXiv: 1008.4884] [INSPIRE].

[114] A. Efrati, A. Falkowski and Y. Soreq, Electroweak constraints on flavorful effective theories, JHEP 07 (2015) 018 [arXiv: 1503.07872] [INSPIRE].

[115] A. Falkowski and K. Mimouni, Model independent constraints on four-lepton operators, JHEP 02 (2016) 086 [arXiv:1511.07434] [INSPIRE].

[116] V. Cirigliano, M. Gonzalez-Alonso and M.L. Graesser, Non-standard charged current interactions: $\beta$ decays versus the LHC, JHEP 02 (2013) 046 [arXiv:1210 .4553] [INSPIRE].

[117] J. de Blas, M. Chala and J. Santiago, Global constraints on lepton-quark contact interactions, Phys. Rev. D 88 (2013) 095011 [arXiv:1307.5068] [INSPIRE].

[118] Z. Han and W. Skiba, Effective theory analysis of precision electroweak data, Phys. Rev. D 71 (2005) 075009 [hep-ph/0412166] [INSPIRE].

[119] L. Berthier and M. Trott, Consistent constraints on the standard model effective field theory, JHEP 02 (2016) 069 [arXiv: 1508.05060] [INSPIRE].

[120] I. Brivio, J. Gonzalez-Fraile, M.C. Gonzalez-Garcia and L. Merlo, The complete HEFT Lagrangian after the LHC Run I, Eur. Phys. J. C 76 (2016) 416 [arXiv:1604.06801] [INSPIRE].

[121] R. Contino, A. Falkowski, F. Goertz, C. Grojean and F. Riva, On the validity of the effective field theory approach to SM precision tests, JHEP 07 (2016) 144 [arXiv: 1604.06444] [INSPIRE].

[122] CMS collaboration, Search for physics beyond the standard model in final states with a lepton and missing transverse energy in proton-proton collisions at $\sqrt{s}=8 \mathrm{TeV}$, Phys. Rev. D 91 (2015) 092005 [arXiv: 1408.2745] [INSPIRE].

[123] A.D. Martin, W.J. Stirling, R.S. Thorne and G. Watt, Parton distributions for the LHC, Eur. Phys. J. C 63 (2009) 189 [arXiv:0901.0002] [InSPIRE]. 